\title{
Modeling and Robust Trajectory Tracking Control for a Novel Six-Rotor Unmanned Aerial Vehicle
}

\author{
Chengshun Yang, ${ }^{1}$ Zhong Yang, ${ }^{1}$ Xiaoning Huang, ${ }^{2}$ Shaobin Li, ${ }^{1}$ and Qiang Zhang ${ }^{1,3}$ \\ ${ }^{1}$ College of Automation Engineering, Nanjing University of Aeronautics and Astronautics, Nanjing 210016, China \\ ${ }^{2}$ School of Electric Power Engineering, Nanjing Institute of Technology, Nanjing 211167, China \\ ${ }^{3}$ School of Electrical Engineering, University of Jinan, Jinan 250022, China \\ Correspondence should be addressed to Zhong Yang; yangzhong@nuaa.edu.cn
}

Received 14 March 2013; Accepted 16 May 2013

Academic Editor: Rongni Yang

Copyright (c) 2013 Chengshun Yang et al. This is an open access article distributed under the Creative Commons Attribution License, which permits unrestricted use, distribution, and reproduction in any medium, provided the original work is properly cited.

\begin{abstract}
Modeling and trajectory tracking control of a novel six-rotor unmanned aerial vehicle (UAV) is concerned to solve problems such as smaller payload capacity and lack of both hardware redundancy and anticrosswind capability for quad-rotor. The mathematical modeling for the six-rotor UAV is developed on the basis of the Newton-Euler formalism, and a second-order sliding-mode disturbance observer (SOSMDO) is proposed to reconstruct the disturbances of the rotational dynamics. In consideration of the under-actuated and strong coupling properties of the six-rotor UAV, a nested double loops trajectory tracking control strategy is adopted. In the outer loop, a position error PID controller is designed, of which the task is to compare the desired trajectory with real position of the six-rotor UAV and export the desired attitude angles to the inner loop. In the inner loop, a rapidconvergent nonlinear differentiator (RCND) is proposed to calculate the derivatives of the virtual control signal, instead of using the analytical differentiation, to avoid "differential expansion" in the procedure of the attitude controller design. Finally, the validity and effectiveness of the proposed technique are demonstrated by the simulation results.
\end{abstract}

\section{Introduction}

In the past years, unmanned aerial vehicles (UAVs) are broadly concerned and applied. They provide tremendous advantages over manned operations like searching and rescuing, remote inspecting, and surveillance as well as in civil use such as disaster monitoring, aerial photographing, and transmission lines inspection. The merit of UAVs is maximized for the practical use in the places where are dangerous and difficult to approach.

UAVs are classified into two categories, fixed and rotary wing types. In practical operations, fixed-wing UAVs have been used for years in routine surveillance missions, but they lack the hovering flight capability. So, a kind of rotary wing type UAVs is focused to offer the possibility of vertical take-off and landing (VOTL), omnidirectional flying, and hovering performance. Rotary wing type UAVs are classified into multirotor type (such as quad-rotor and six-rotor), coaxial helicopter, traditional helicopter, and so forth. quadrotor has the simplest mechanical structure among them. Moreover, its simple design and relatively low-cost feature make it an attractive candidate for swarm operations, a field of ongoing research in the UAV community [1-5].

However, quad-rotor also has some potential defects such as smaller payload capacity, lack of hardware redundancy, and anticrosswind capability. For this reason, we introduce, in this paper, one configuration of a six-rotor UAV (the structure as shown in Figure 1) composed of six rotors to solve these problems of quad-rotor. The main characteristics of the sixrotor UAV are increased payload capacity and stability in the windy and hardware redundancy. And the six rotors and propellers of the UAV are distributed on both sides of the fuselage symmetrically. So, the configuration of the sixrotor UAV is similar to an insect. The detailed structure, features, and purpose of the six-rotor UAV can be seen in $[6,7]$. 
Compared with the quad-rotor, the super characteristics of the six-rotor UAV are as follows.

(1) Increased Payload Capability. By the actual flight experiment, the six-rotor UAV has a payload capacity of approximately 0.9 kilogram, the largest of all the quad-rotor with the same mechanical size.

(2) Increased Stability in the Wind. The six-rotor UAV weights approximately 1700 grams and has the increased thrust of 6 counter-rotating rotors which allows it to be much more stable in windy conditions. The lager size and weight of the six-rotor UAV make it a very stable UAV to fly.

(3) Hardware Redundancy and Fault Tolerance. Six-rotor UAV could remain stable flighting while some of the six rotors are broken. If one or two rotors are broken, the flight controller with fault diagnosis and fault-tolerant can implement fault diagnosis and fault tolerant flight control immediately, and other rotors will compensate the reduced lift caused by the broken rotors to keep the stability of the six-rotor UAV. It can effectively improve the reliability and safety of the UAV and reduce the probability of crash. As for the quad-rotor, it will crash inevitably in the above situations.

At the same time, in this paper, the translational dynamics and rotational dynamics with aerodynamic moments, external disturbance, and parameter uncertainty of the six-rotor UAV are also developed. And, a second-order sliding-mode disturbance observer (SOSMDO) is proposed to reconstruct the disturbance of the rotational dynamics.

Next, a robust trajectory tracking control strategy forcing the six-rotor UAV to track the desired trajectory accurately is designed. Considering the under-actuated and strong coupling characteristics of the six-rotor UAV, the closed loop flight control system is treated as a nested doubleloops structure system, where the outer loop indicates the relationship between translation and rotation movement, and the inner loop denotes the attitude control loop. Meanwhile, in the outer loop, a position error PID controller is developed, of which the task is to compare the desired trajectory with real position of the six-rotor UAV and construct the desired attitude angles to the inner attitude control loop. In the control methods of nonlinear system, fuzzy variable structure control [8], network-based feedback control [9], dynamic inversion [10], feed linearization and sliding mode control [11], and adaptive output feedback control [12] have been widely used. Backstepping control design [3-5] has also become an effective approach for designing controller because of its simplicity. The rotational dynamics of the six-rotor UAV satisfies the strict feedback form, so backstepping technique can be used to design the attitude controller. However, the analytic derivative expressions of virtual control variables are usually overly complicated or unknown especially for the uncertain systems, which limits the backstepping technique in practical applications. Therefore, to overcome this drawback, a rapidconvergent nonlinear differentiator (RCND) is proposed to extract the ideal angular rate differential command signals and without calculating the virtual control signal derivative

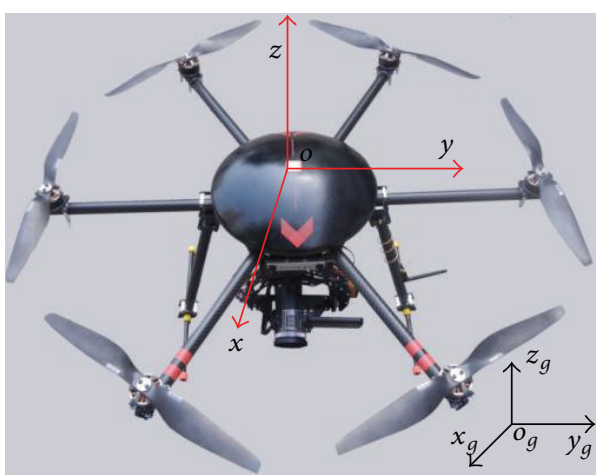

FIGURE 1: The structure of six-rotor UAV and the associated frames.

analytically, and to decrease the dependent degree on the analytic model. In succession, an attitude controller of the sixrotor UAV based on disturbances compensation, RCDN and backstepping is designed. And the robust terms of the attitude controller can reduce the effect of disturbance reconstruction errors on the tracking capability of the six-rotor UAV. Finally, the validity and effectiveness of the proposed SOSMDO and trajectory tracking strategy are demonstrated through various simulation experiments.

The whole paper is organized as follows. Section 2 presents the detailed mathematical model of the six-rotor UAV. Section 3 presents a second-order sliding-mode disturbance observer for disturbance reconstruction. The robust trajectory tracking control strategy is proposed in Section 4. The position controller and attitude stability controller are designed, respectively, in this section. In Section 5, simulation results of the six-rotor UAV trajectory tracking are compared and discussed. And finally, the conclusion of the proposed robust trajectory control strategy is presented in Section 6.

\section{Mathematical Modeling}

In order to simplify the modeling of the six-rotor UAV and therefore make the controller design easier, several reasonable assumptions are made.

Assumption 1. Six-rotor UAV is rigid. Then, the nonlinear dynamics can be derived by using Newton-Euler formulas.

Assumption 2. The configuration of the six-rotor UAV is symmetrical with respect to the axes $o x, o y$, and $o z$.

Assumption 3. The height between the rotors and the plane of the six-rotor UAV is ignored.

Firstly, two frames have to be defined: a body-fixed frame $(\mathbb{B}$-frame) and an earth-fixed inertial frame ( $\mathbb{E}$-frame). Let $\mathbb{B}=\{$ oxyz $\}$ denote the body-fixed frame whose origin $o$ is at the center of mass of the six-rotor UAV and $\mathbb{E}=\left\{o_{g} x_{g} y_{g} z_{g}\right\}$ denote the earth-fixed inertial frame, as shown in Figure 1. Therefore, under Assumption 3, the structure of the six-rotor UAV can be simplified as shown in Figure 2. 


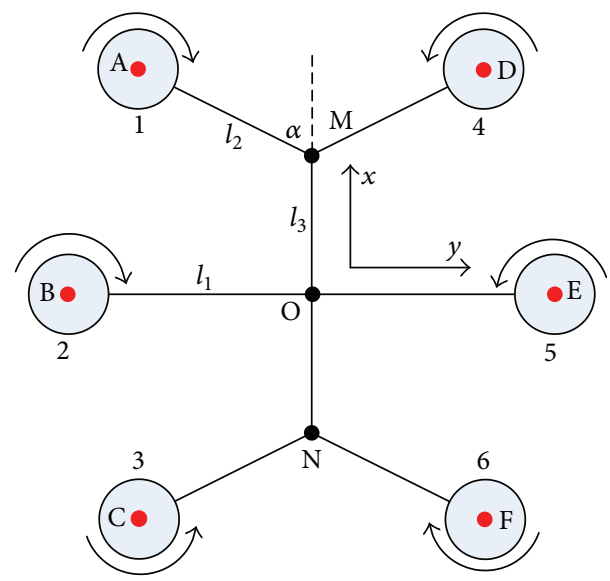

FIgURE 2: The simple structure of six-rotor UAV.

In Figure $2, l_{1}$ denotes the length of $\mathrm{OB}$, and $\mathrm{OE}, l_{2}$ denotes the length of AM, DM, CN, and $\mathrm{FN} ; l_{3}$ denotes the length of $\mathrm{OM}$ and $\mathrm{ON}$, and $\alpha$ represents the included angle between $\mathrm{AM}$ and $\mathrm{OM}$.

2.1. Translational Dynamics. Modeling the rigid body dynamics aims at finding the differential equations that relate system outputs (position and orientation) to its inputs (force and torque vectors). The equations of motion for a rigid body of mass $m \in R$ and inertial matrix $\mathbf{J} \in R^{3 \times 3}$ subject to extern force vector $\mathbf{F} \in R^{3}$ and torque vector $\tau^{b} \in R^{3}$ are given by the following Newton-Euler formulas [14], expressed in the body-fixed frame $\mathbb{B}$ :

$$
\begin{aligned}
& m \dot{\mathbf{V}}+\Omega \times m \mathbf{V}=\mathbf{F}, \\
& \mathbf{J} \dot{\Omega}+\Omega \times \mathbf{J} \Omega=\tau^{b},
\end{aligned}
$$

where $\mathbf{V}=(u, v, w)^{T}$ and $\Omega=(p, q, r)^{T}$ are the linear and angular velocities in the body-fixed frame, respectively. The translational force $\mathbf{F}$ combines gravity, main thrust, and other body forces components. Under Assumption 2, the total inertial matrix $\mathbf{J} \in R^{3 \times 3}$ of the six-rotor UAV is a diagonal positive definite constant matrix expressed in the body-fixed frame $\mathbb{B}$.

Using Euler angles parametrization and " $Z Y X$ " convention, the airframe orientation in space is given by a rotation matrix $\mathbf{R}(\phi, \theta, \psi)$ from the body-fixed frame $\mathbb{B}$ to the earthfixed inertial frame $\mathbb{E}$, where $\mathbf{R} \in S O(3)$ is given by

$$
\mathbf{R}=\left[\begin{array}{ccc}
\cos \theta \cos \psi & \sin \theta \cos \psi \sin \phi-\sin \psi \cos \phi & \sin \theta \cos \psi \cos \phi+\sin \psi \sin \phi \\
\cos \theta \sin \psi & \sin \theta \sin \psi \sin \phi+\cos \psi \cos \phi & \sin \theta \sin \psi \cos \phi-\cos \psi \sin \phi \\
-\sin \theta & \cos \theta \sin \phi & \cos \theta \cos \phi
\end{array}\right],
$$

where $\xi=(\phi, \theta, \psi)^{T}$ denotes the vector of three Euler angles.

By considering this transformation between the bodyfixed frame $\mathbb{B}$ and the earth-fixed inertial frame $\mathbb{E}$, it is possible to separate the gravitational force from other force and write the translational dynamics in the earth-fixed inertial frame as follows:

$$
\begin{gathered}
\dot{\mathbf{p}}=\mathbf{v}, \\
\dot{\mathbf{v}}=-g \mathbf{z}_{e}+\frac{1}{m} \mathbf{R} \mathbf{F}^{b},
\end{gathered}
$$

where $\mathbf{p}=(x, y, z)^{T}$ and $\mathbf{v}=(\dot{x}, \dot{y}, \dot{z})^{T}$ are the position and velocity of the six-rotor UAV in the inertial frame, respectively. $g$ is the gravitational acceleration, $\mathbf{F}^{b}=(0,0, T)^{T}$ is the resulting force vector in the body-fixed frame (excluding the gravity force) acting on the airframe, and $\mathbf{z}_{e}=(0,0,1)^{T}$ is a unit vector expressed in the earth-fixed inertial frame.

2.2. Rotational Dynamics. In the following, we will use Euler angles parametrization to express the rotational dynamics in an appropriate form for control design.

Let us first write the kinematic relation between $\Omega$ and $\dot{\xi}$ :

$$
\dot{\xi}=\mathrm{W} \Omega \text {, }
$$

where the Euler matrix $\mathbf{W}$ is given by

$$
\mathbf{W}=\left[\begin{array}{ccc}
1 & \sin \phi \tan \theta & \cos \phi \tan \theta \\
0 & \cos \phi & -\sin \phi \\
0 & \sin \phi \sec \theta & \cos \phi \sec \theta
\end{array}\right]
$$

and $\operatorname{det}(\mathbf{W})=\sec \theta$. Thus, when the pitch angle satisfies $\theta \neq(2 k-1) \pi / 2, k \in Z$ the matrix $\mathbf{W}$ is invertible.

Therefore, from (4) and the second equation of (1), the rotational dynamics of the six-rotor UAV, used for flight controller, is given by

$$
\begin{gathered}
\dot{\xi}=\mathbf{W} \Omega+\mathbf{D}_{\xi}, \\
(\mathbf{J}+\Delta \mathbf{J}) \dot{\Omega}=-[\Omega \times(\mathbf{J}+\Delta \mathbf{J}) \Omega]-\mathbf{G}_{a}+\tau^{b}+\mathbf{d},
\end{gathered}
$$

where (6) expresses the attitude angle loop and angular rate loop of the six-rotor UAV, respectively. And $\mathbf{D}_{\xi}$ is an external disturbance of the attitude angle loop. $\Delta \mathbf{J} \in R^{3 \times 3}$ denotes an uncertain part of the inertial matrix, which is caused by variations of particular payloads from a nominal one. $\mathbf{d}=$ $\left[d_{\phi}, d_{\theta}, d_{\psi}\right]^{T}$ denotes the aerodynamic moments and external 
disturbance. And the vector $\mathbf{G}_{a}$ expresses the gyroscopic torque which is given by

$$
\mathbf{G}_{a}=J_{r}\left(\Omega \times \mathbf{z}_{e}\right)\left(\sum_{i=3,4,5} \omega_{i}-\sum_{i=1,2,6} \omega_{i}\right),
$$

where $J_{r}$ and $\omega_{i}$ are, respectively, the rotor inertia and the rotor speed.

For the simplicity of this study, the rotational dynamics (6) for the six-rotor UAV with aerodynamic moments, external disturbance, and parameter uncertainty input is rewritten as

$$
\begin{gathered}
\dot{\xi}=\mathbf{W} \Omega+\mathbf{D}_{\xi}, \\
\dot{\Omega}=-\mathbf{J}^{-1}(\Omega \times \mathbf{J} \Omega)-\mathbf{J}^{-1} \mathbf{G}_{a}+\mathbf{J}^{-1} \tau^{b}+\mathbf{D}_{\Omega},
\end{gathered}
$$

where $\mathbf{D}_{\Omega}=\mathbf{J}^{-1}[\mathbf{d}-\Delta \mathbf{J} \dot{\Omega}-(\Omega \times \Delta \mathbf{J} \Omega)]$ is called the composite disturbance of the angular rate loop, (9), which includes aerodynamic moments, external disturbance, and parameter uncertainty.

The expressions of $\mathbf{F}^{b}$ of (3) and $\tau^{b}$ of (9) are detailed in the next section.

2.3. Aerodynamics Force and Torques. The six-rotor UAV is under-actuated mechanical system with six degree of freedom (DOF) and four main control inputs. It is characterized by three main control torques $\tau^{b}=\left(\tau_{\phi}, \tau_{\theta}, \tau_{\psi}\right)^{T}$ and one main control force $\mathbf{F}^{b}=(0,0, T)^{T}$. The collective lift $T$ is the sum of the thrusts generated by the six propellers in the free air:

$$
T=\sum_{i=1}^{6} f_{i}=b \sum_{i=1}^{6} \omega_{i}^{2}
$$

At the same time, the reactive torque caused by air drag is also generated by each propeller and given by $Q_{i}=d \omega_{i}^{2}$. Thus, the total reactive torque by the six propellers is given by

$$
Q=\sum_{i=3,4,5} Q_{i}-\sum_{i=1,2,6} Q_{i}=d\left(\sum_{i=3,4,5} \omega_{i}^{2}-\sum_{i=1,2,6} \omega_{i}^{2}\right),
$$

where $\omega_{i}$ is the rotor speed. The parameters $b$ and $d$ are positive proportionality constants relative to the density of air, the shape of the blades, the number of the blades, the chord length of the blades, the pitch angle of the blade airfoil, and the drag constant. And it is reasonable to assume that the scalars $b$ and $d$ are constants when the six-rotor UAV is performed at low speed in free air [4].

Therefore, according to Figure 2 and (11), we can easily obtain that the airframe torques generated by the six rotors are given by

$$
\begin{aligned}
\tau^{b} & =\left[\begin{array}{c}
\tau_{\phi} \\
\tau_{\theta} \\
\tau_{\psi}
\end{array}\right] \\
& =\left[\begin{array}{c}
b l_{2} \sin \alpha\left(\omega_{4}^{2}+\omega_{6}^{2}-\omega_{1}^{2}-\omega_{3}^{2}\right)+b l_{1}\left(\omega_{5}^{2}-\omega_{2}^{2}\right) \\
b\left(l_{2} \cos \alpha+l_{3}\right)\left(\omega_{1}^{2}+\omega_{4}^{2}-\omega_{3}^{2}-\omega_{6}^{2}\right) \\
d\left(\omega_{3}^{2}+\omega_{4}^{2}+\omega_{5}^{2}-\omega_{1}^{2}-\omega_{2}^{2}-\omega_{6}^{2}\right)
\end{array}\right] .
\end{aligned}
$$

In order to facilitate the computation of the real control inputs, that is, $\omega_{i},(i=1,2, \ldots, 6),(10)$ and $\tau^{b}$ put together

$\left.\begin{array}{ccc}b & b & b \\ b l_{2} \sin \alpha & b l_{1} & b l_{2} \sin \alpha \\ \left.d l_{2} \cos \alpha+l_{3}\right) & 0 & b\left(l_{2} \cos \alpha+l_{3}\right) \\ d & d & -d\end{array}\right]\left[\begin{array}{c}\omega_{1}^{2} \\ \omega_{2}^{2} \\ \omega_{3}^{2} \\ \omega_{4}^{2} \\ \omega_{5}^{2} \\ \omega_{6}^{2}\end{array}\right]=\mathbf{M} \bar{\omega}$.

\section{SOSMDO Design}

Sliding mode disturbance observer (SMDO) has advantages of easy realization, simple design, rapid convergence, and so forth. Besnard et al. [15] uses SMDO to reconstruct the disturbance of the mathematical model of quad-rotor. But in [15], the disturbance reconstruction terms all contain symbolic function, and it will cause discontinuity of the disturbance compensation and chattering phenomenon. To solve this problem, Zeng et al. [16] design an SMDO based on super-twisting algorithm to reconstruct and compensate the disturbance of a missile system. The algorithm can make the high frequency switching part hidden into the higher derivative of the sliding mode variable, and thus the chattering phenomena can be weakened. However, the reconstruction terms of the algorithm still contain symbolic function, and the algorithm is relatively complicated.

In view of this, in this paper, a second-order sliding mode disturbance observer (SOSMDO) to provide continuous disturbance compensation term is introduced which makes use of the integral of a symbolic function to reconstruct 
the disturbance. Compared with the algorithm in [16], the proposed SOSMDO has the advantage of implementing easily, designing process simply, and inhibiting chattering phenomenon further.

First, in order to make the SOSMDO design easy, a couple of reasonable assumptions are given as follows.

Assumption 4. The states $\xi$ and $\Omega$ of the system (8) and (9) are accessible for measurement.

Assumption 5. Partial derivatives with respect to time of the disturbances $\mathbf{D}_{i}, i=\xi, \Omega$ are continuous and bounded, and there exists a known bounded constant $C_{i}$ such that

$$
\left\|\alpha_{i}(\cdot)\right\|_{\infty}=\sup _{t \in[0, \infty)}\left\|\frac{\partial \mathbf{D}_{i}}{\partial t}\right\| \leq C_{i} \quad(i \in \xi, \Omega) .
$$

Theorem 6. Consider the rotational dynamics of the six-rotor $U A V$ (8) and (9) under Assumptions 4 and 5; consider the following second-order sliding mode dynamics:

$$
\begin{gathered}
\mathbf{s}_{i}=x_{i}-z_{i}, \\
\dot{\mathbf{z}}_{i}=\Gamma_{i} s_{i}+\varphi_{i}+\widehat{\mathbf{D}}_{i}, \\
\dot{\widehat{\mathbf{D}}}_{i}=\chi_{i} \operatorname{sign}\left(s_{i}\right),
\end{gathered}
$$

where $i \in\{\xi, \Omega\}, \mathbf{s}_{i}=\left[\begin{array}{lll}s_{i 1} & s_{i 2} & s_{i 3}\end{array}\right]^{T} \in R^{3}$ is an auxiliary sliding vector, $\Gamma_{i}=\operatorname{diag}\left\{k_{i 1}, k_{i 2}, k_{i 3}\right\} \in R^{3 \times 3}$ is a positive definite matrix to be designed if $i=\xi, \varphi_{\xi}=\mathrm{W} \Omega$ and if $i=\Omega, \varphi_{\Omega}=$ $-\mathbf{J}^{-1}(\Omega \times \mathbf{J} \Omega)-\mathbf{J}^{-1} \mathbf{G}_{a}+\mathbf{J}^{-1} \tau_{a} . \widehat{\mathbf{D}}_{i}$ is the disturbance reconstruction value, and $\operatorname{sign}\left(s_{i}\right)=\left[\begin{array}{lll}\operatorname{sign}\left(s_{i 1}\right) & \operatorname{sign}\left(s_{i 2}\right) & \operatorname{sign}\left(s_{i 3}\right)\end{array}\right]^{T}$.

When we choose $\chi_{i}>C_{i}$, the auxiliary sliding vector $\mathbf{s}_{i}$ and its derivative $\dot{\mathbf{s}}_{i}$ converge to origin asymptotically.

Proof. Substituting (8) and (9) into (15), respectively, we have

$$
\dot{\mathbf{s}}_{i}=-\Gamma_{i} \mathbf{s}_{i}+\mathbf{D}_{i}-\widehat{\mathbf{D}}_{i} \quad i \in\{\xi, \Omega\} .
$$

And under Assumption 5, taking the time derivative of (16) and substituting it into (15), it yields that

$$
\ddot{\mathbf{s}}_{i}+\Gamma_{i} \dot{\mathbf{s}}_{i}=-\eta_{i} \operatorname{sign}\left(s_{i}\right)
$$

where $\eta_{i} \in\left[\chi_{i}-C_{i}, \chi_{i}+C_{i}\right]$.

Select the candidate Lyapunov function as

$$
V_{i}=\sum_{j=1}^{3} \frac{1}{2}\left[s_{i j}, \dot{s}_{i j}\right] \mathbf{Q}_{i j}\left[s_{i j}, \dot{s}_{i j}\right]^{T}+p_{i j} \eta \int_{0}^{s_{i j}} \operatorname{sign}\left(s_{i j}\right) d s_{i j}
$$

where $\mathbf{Q}_{i j}=\left[\begin{array}{ll}q_{i j 1} & q_{i j 3} \\ q_{i j 3} & q_{i j 2}\end{array}\right]$ is a positive definite matrix to be designed and it satisfies $q_{i j 1} q_{i j 2}>q_{i j 3}^{2}, q_{i j 1}=k_{i j} q_{i j 3}, q_{i j 2}=p_{i j}$, $q_{i j 2} k_{i j}>q_{i j 3}$.
Then, substituting the positive definite parameter matrix $\mathbf{Q}_{i j}$ and (17) into the time derivative of (18) yields that

$$
\begin{aligned}
\dot{V}_{i}= & \sum_{j=1}^{3}\left[\dot{s}_{i j}, \ddot{s}_{i j}\right] \mathbf{Q}_{i j}\left[s_{i j}, \dot{s}_{i j}\right]^{T}+p_{i j} \eta \dot{s}_{i j} \operatorname{sign}\left(s_{i j}\right) \\
= & \sum_{j=1}^{3} q_{i j 1} s_{i j} \dot{s}_{i j}+\left(q_{i j 2} \dot{s}_{i j}+q_{i j 3} s_{i j}\right)\left(-k_{i j} \dot{s}_{i j}-\eta \operatorname{sign}\left(s_{i j}\right)\right) \\
& +q_{i j 3} \dot{s}_{i j}^{2}+p_{i j} \eta \dot{s}_{i j} \operatorname{sign}\left(s_{i j}\right) \\
= & \sum_{j=1}^{3}-\left(k_{i j} q_{i j 2}-q_{i j 3}\right) \dot{s}_{i j}^{2}-\eta q_{i j 3} s_{i j} \operatorname{sign}\left(s_{i j}\right) .
\end{aligned}
$$

From (19), we can get the conclusion that if $s_{i j} \neq 0$ or $\dot{s}_{i j} \neq 0$, then $\dot{V}_{i}<0$ is true. Thus, the auxiliary sliding vector $\mathbf{s}_{i}$ and its derivative $\dot{\mathbf{s}}_{i}$ of the SOSMDO converge to origin asymptotically.

This ends the proof.

Remark 7. Compared with the disturbance reconstruction based on SMDO in [16], the proposed SOSMDO in this paper, the disturbance reconstruction vectors are continuous, and the high frequency switching part is hidden into the first-order derivative of the sliding mode variable. Therefore, the SOSMDO can remarkably restrain the chattering phenomenon.

\section{Robust Trajectory Tracking Control Strategy}

To give a clear idea of the robust trajectory tracking control strategy proposed in this paper, a control block diagram is depicted in Figure 4. The outer loop controller employs PID control methodology to compare the desired trajectory with real position of the six-rotor UAV and construct the desired attitude angles to the inner attitude control loop. In the inner loop, a robust attitude controller based on disturbance compensation and RCND is designed in which uses a nonlinear differentiator to calculate the derivative of the virtual control signal, instead of using the analytical differentiation.

4.1. Translational Control. Define the position tracking error $\mathbf{p}_{e}$ of the translational dynamics given by (3) as

$$
\mathbf{p}_{e}=\mathbf{p}_{c}-\mathbf{p}=\left(x_{c}-x, y_{c}-y, z_{c}-z\right)^{T},
$$

where the vector $\mathbf{p}_{c}=\left(x_{c}, y_{c}, z_{c}\right)^{T}$ is the desired smooth trajectory.

A sliding mode error function is defined as follows [17]:

$$
\mathbf{r}=\dot{\mathbf{p}}_{e}+\Lambda \mathbf{p}_{e}
$$

where $\Lambda$ is a diagonal positive definite design parameter matrix. Then, (21) is a stable system so that $\mathbf{p}_{e}$ is bounded as 


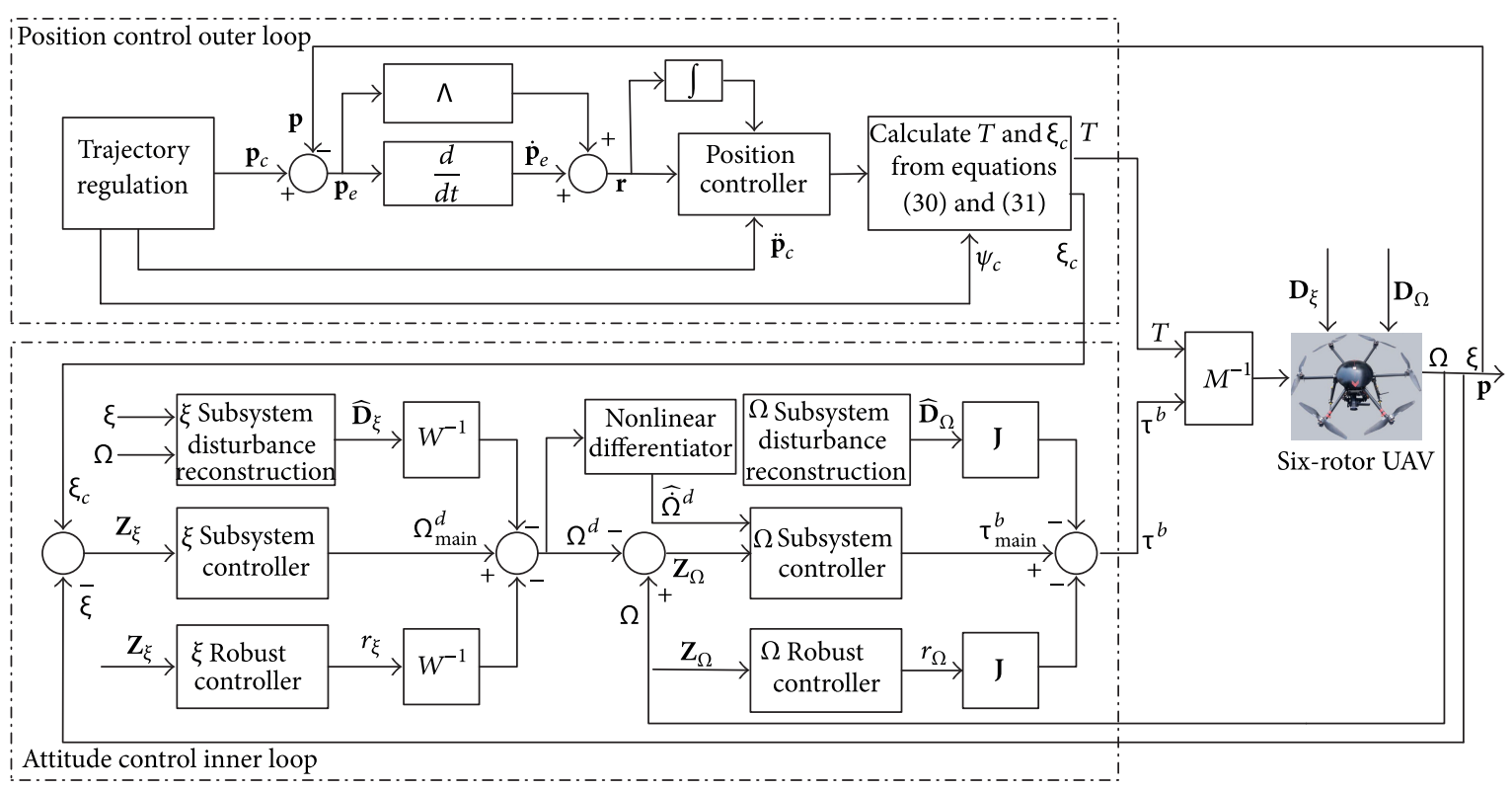

FIGURE 3: Block diagram of the robust trajectory tracking control strategy.
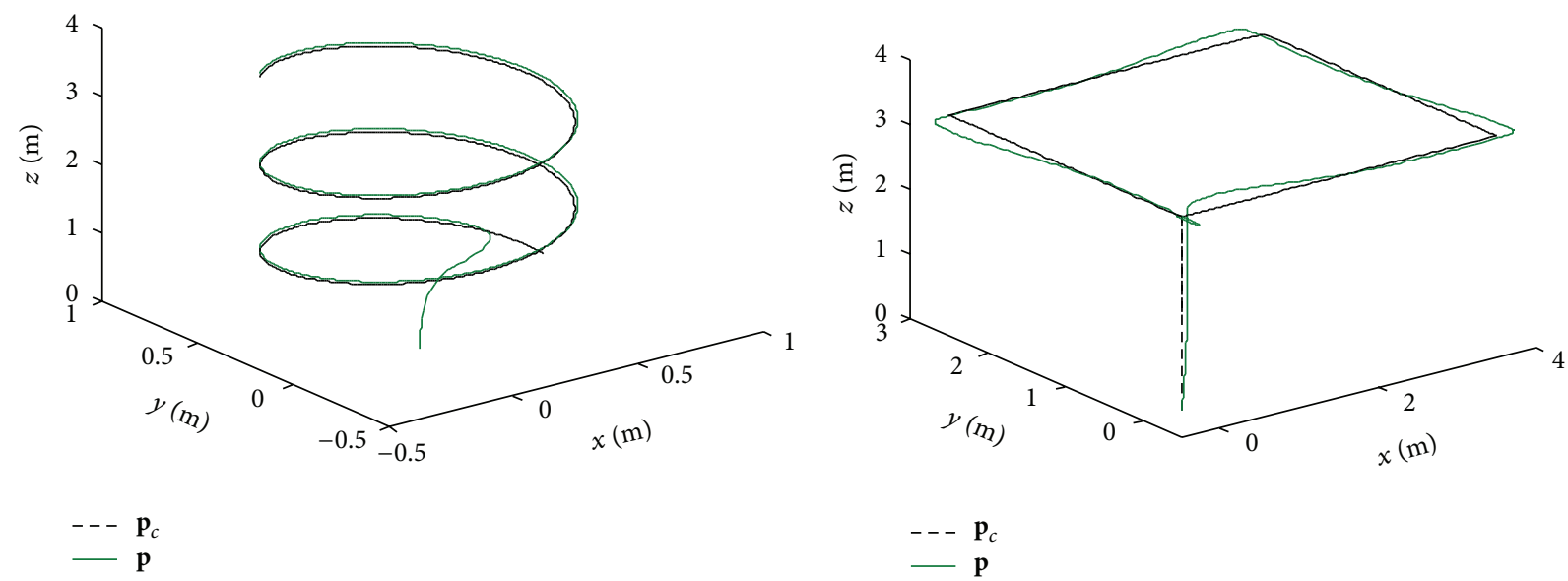

(a) Vertical helix flight

(b) Horizontal rectangle flight

FIGURE 4: The 3D diagram for real flight trajectory (solid line) and desired trajectory (dashed line).

long as the controller guarantees that the filtered error $\mathbf{r}$ is bounded. In fact, it is easy to show that one has [17]

$$
\left\|\mathbf{p}_{e}\right\| \leq \frac{\|\mathbf{r}\|}{\lambda_{\min }(\Lambda)}, \quad\left\|\dot{\mathbf{p}}_{e}\right\| \leq\|\mathbf{r}\|,
$$

where $\lambda_{\min }(\Lambda)$ is the smallest eigenvalue of the matrix $\Lambda$.

Note that $\dot{\mathbf{p}}_{e}+\Lambda \mathbf{p}_{e}=0$ defines a stable sliding mode surface. The function of the controller to be designed is to force the system onto this surface by making $\mathbf{r}$ small. The parameter $\Lambda$ is selected for a desired sliding mode response

$$
\mathbf{p}_{e}(t)=\mathbf{p}_{e}^{-\Lambda t} \mathbf{p}_{e}(0) .
$$

We now focus on designing a controller to keep $\|\mathbf{r}\|$ small. Then, the error dynamics becomes

$$
m \dot{\mathbf{r}}=m \ddot{\mathbf{p}}_{e}+m \Lambda \dot{\mathbf{p}}_{e}
$$

Then, we can obtain

$$
m \mathbf{r}=m\left(\begin{array}{c}
\ddot{x}_{c} \\
\ddot{y}_{c} \\
\ddot{z}_{c}
\end{array}\right)-m\left(\begin{array}{c}
\ddot{x} \\
\ddot{y} \\
\ddot{z}
\end{array}\right)+m \Lambda\left(\mathbf{r}-\Lambda \mathbf{p}_{e}\right) .
$$

Submitting the second equation of (3) into (25), we have

$$
m \dot{\mathbf{r}}=m \ddot{\mathbf{p}}_{e}-\mathbf{U}-\mathbf{m}_{g}+m \Lambda \mathbf{r}-m \Lambda^{2} \mathbf{p}_{e},
$$


where

$$
\begin{gathered}
\mathbf{U}=\left[\begin{array}{c}
T(\sin \theta \cos \psi \cos \phi+\sin \psi \sin \phi) \\
T(\sin \theta \sin \psi \cos \phi-\cos \psi \sin \phi) \\
T \cos \theta \cos \phi
\end{array}\right], \\
\mathbf{m}_{g}=\left[\begin{array}{c}
0 \\
0 \\
-m g
\end{array}\right] .
\end{gathered}
$$

Then, the virtual control input $\widehat{\mathbf{U}}=\left(u_{x}, u_{y}, u_{z}\right)^{T}$ which stabilizes the tracking error dynamics (26) is designed as

$$
\widehat{U}=m \ddot{\mathbf{p}}_{e}-m \Lambda^{2} \mathbf{p}_{e}-\mathbf{m}_{g}+\mathbf{K}_{r} \mathbf{r}+\mathbf{K}_{i} \int_{0}^{t} \mathbf{r} d t^{\prime},
$$

where $\mathbf{K}_{r}$ and $\mathbf{K}_{i}$ are diagonal positive definite matrices to be designed.

Now, let us computer the control force vector magnitude and the desired attitude angles for tracking the desired trajectory. The virtual control input $\widehat{\mathbf{U}}=\left(u_{x}, u_{y}, u_{z}\right)^{T}$ calculated by the (28) is the force vector components along axes that are needed for tracking some desired trajectory. Thus, the components of $\widehat{\mathbf{U}}$ are given by

$$
\begin{gathered}
u_{x}=T\left(\sin \theta_{c} \cos \psi_{c} \cos \phi_{c}+\sin \psi_{c} \sin \phi_{c}\right), \\
u_{y}=T\left(\sin \theta_{c} \sin \psi_{c} \cos \phi_{c}-\cos \psi_{c} \sin \phi_{c}\right), \\
u_{z}=T \cos \theta_{c} \cos \phi_{c} .
\end{gathered}
$$

According to (29), the required total thrust $T$ and the desired attitude angles $\phi_{c}$ and $\theta_{c}$ can be proposed as

$$
\begin{gathered}
T=\sqrt{u_{x}^{2}+u_{y}^{2}+u_{z}^{2}} \\
\phi_{c}=\arcsin \left(\frac{u_{x} \sin \psi_{c}-u_{y} \cos \psi_{c}}{\sqrt{u_{x}^{2}+u_{y}^{2}+u_{z}^{2}}}\right) \\
\theta_{c}=\arctan \left(\frac{u_{x} \cos \psi_{c}+u_{y} \sin \psi_{c}}{u_{z}}\right),
\end{gathered}
$$

where $\psi_{c}$ is the desired yaw angle given by as an external reference command.

Theorem 8. Consider the translational dynamics of the sixrotor as described in (3) with a control strategy shown in Figure 3 and control input given by (28) and (30). And suppose that $\left[\mathbf{K}_{r}-m \Lambda\right]>0$. Then, the error $\mathbf{r}$ and the position tracking error $\mathbf{p}_{e}$ are uniformly ultimately bounded (UUB).

Proof. Using (28) into (26) yields the closed-loop error dynamics

$$
m \dot{\mathbf{r}}=-\left(\mathbf{K}_{r}-m \Lambda\right) \mathbf{r}-\mathbf{K}_{i} \int_{0}^{t} \mathbf{r} d t^{\prime}
$$

Choose the candidate Lyapunov function as

$$
L=\frac{1}{2} \mathbf{r}^{T} m \mathbf{r}+\frac{1}{2}\left[\int_{0}^{t} \mathbf{r}^{T} d t^{\prime}\right] \mathbf{K}_{i}\left[\int_{0}^{t} \mathbf{r} d t^{\prime}\right],
$$

and the time derivative of $L$ with respect to time is given by

$$
\dot{L}=\mathbf{r}^{T} m \dot{\mathbf{r}}+\mathbf{r}^{T} \mathbf{K}_{i} \int_{0}^{t} \mathbf{r} d t^{\prime}
$$

Substituting (32) into (34), we obtain

$$
\dot{L}=-\mathbf{r}^{T}\left(\mathbf{K}_{r}-m \Lambda\right) \mathbf{r} \leq-\left\|\mathbf{K}_{r}-m \Lambda\right\|_{\min }\|\mathbf{r}\|^{2} \leq 0,
$$

where $\left\|\mathbf{K}_{r}-m \Lambda\right\|_{\min }$ is the minimum possible norm of different parameter values of $\left(\mathbf{K}_{r}-m \Lambda\right)$.

Then, we conclude that the error $\mathbf{r}$ is UUB. Moreover, according to the definition of $\mathbf{r}$ given by (21), we have

$$
\left\|\mathbf{p}_{e}\right\| \leq\|s+\Lambda\|^{-1}\|\mathbf{r}\| \leq\left(\lambda_{\min }(\Lambda)\right)^{-1}\|\mathbf{r}\|,
$$

where $\lambda_{\min }(\Lambda)$ is the smallest eigenvalue of $\Lambda$.

Thus, from (36), we prove that the position tracking error $\mathbf{p}_{e}$ is also UUB.

\subsection{Rotational Control}

4.2.1. RCND Design. Using the approach of combination of linear and nonlinear differentiator to design the RCND under the introduction of perturbation parameter which is the design idea of the RCND. In order to weaken the chattering of differential estimation, a perturbation parameter is introduced into nonlinear term under the proposed differentiator robustness, and a linear term with perturbation parameter is utilized to accelerate the convergence speed of the differentiator and restrain the high frequency noise interference.

Define $\operatorname{sig}(\mathbf{x})^{m}=\left[\operatorname{sig}\left(x_{1}^{m}\right), \ldots, \operatorname{sig}\left(x_{n}^{m}\right)\right]^{T}$, where $\operatorname{sig}\left(x_{1}^{m}\right)=\left|x_{i}\right|^{m} \operatorname{sign}\left(x_{i}\right), m>0, \mathbf{x} \in R^{n}$, and sign is a symbolic function.

To give a clear idea of the RCND design procedure the following step is given.

Lemma 9. Consider the nonlinear system in the form of

$$
\begin{gathered}
\dot{\mathbf{x}}_{1}(t)=\mathbf{x}_{2}(t), \\
\dot{\mathbf{x}}_{2}(t)=-\mathbf{R}_{1}^{1} \mathbf{x}_{1}(t)-\mathbf{R}_{1}^{2} \operatorname{sig}\left(\mathbf{x}_{1}\right)^{\varepsilon /(2-\varepsilon)}(t)-\mathbf{R}_{2}^{1} \mathbf{x}_{2}(t) \\
-\mathbf{R}_{2}^{2} \operatorname{sig}\left(\mathbf{x}_{2}\right)^{\varepsilon}(t),
\end{gathered}
$$

where $\mathbf{x}_{1}(t), \mathbf{x}_{2}(t) \in R^{n}$ are the states of the nonlinear system. $\mathbf{R}_{1}^{1}=\operatorname{diag}\left\{r_{11}^{1}, \ldots, r_{1 n}^{1}\right\}, \mathbf{R}_{1}^{2}=\operatorname{diag}\left\{r_{11}^{2}, \ldots, r_{1 n}^{2}\right\}, \mathbf{R}_{2}^{2}=$ $\operatorname{diag}\left\{r_{21}^{2}, \ldots, r_{2 n}^{2}\right\}, \mathbf{R}_{2}^{1}>0$ and $0<\varepsilon<1$ are the parameters to be designed.

For any initial bounded $\mathbf{x}_{1}(0)$ and $\mathbf{x}_{2}(0)$, the system (37) converges to origin in a limited time. This means that there exists a time constant such that

$$
\mathbf{x}_{1}(t) \equiv 0, \quad \mathbf{x}_{2}(t) \equiv 0 \quad \forall t \geq t_{f} .
$$

Proof. Based on (37), we choose the following Layapunov function:

$$
\mathbf{V}=\frac{1}{2}\left(\mathbf{x}_{1}^{T} \mathbf{R}_{1}^{1} \mathbf{x}_{1}+\mathbf{x}_{2}^{T} \mathbf{x}_{2}\right)+\sum_{j=1}^{n} \frac{r_{1 j}^{2}(2-\varepsilon)}{2}\left|x_{1 j}\right|^{2 /(2-\varepsilon)} .
$$


The time derivative of $V$ along the trajectories of (37) is given by

$$
\begin{aligned}
\dot{V}= & \mathbf{x}_{1}^{T} \mathbf{R}_{1}^{1} \mathbf{x}_{2} \\
& +\mathbf{x}_{2}^{T}\left[-\mathbf{R}_{1}^{1} \mathbf{x}_{1}-\mathbf{R}_{1}^{2} \operatorname{sig}\left(\mathbf{x}_{1}\right)^{\varepsilon /(2-\varepsilon)}(t)-\mathbf{R}_{2}^{1} \mathbf{x}_{2}-\mathbf{R}_{2}^{2} \operatorname{sig}\left(\mathbf{x}_{2}\right)^{\varepsilon}\right] \\
& +\sum_{j=1}^{n} r_{1 j}^{2} \operatorname{sig}\left(x_{1 j}\right)^{\varepsilon /(2-\varepsilon)} x_{2 j} \\
\leq & -\mathbf{R}_{2}^{1} \mathbf{x}_{2}^{T} \mathbf{x}_{2}-\sum_{j=1}^{n} r_{2 j}^{2}\left|x_{2 j}\right|^{1+\varepsilon}<0 .
\end{aligned}
$$

Under the bounded initial values $\mathbf{x}_{1}(0)$ and $\mathbf{x}_{2}(0)$, the solution of the system (37) can be calculated by [18]. In addition, from the solution of (37) and (40) and LaSalle's Invariant Principle, we conclude that the system (37) is asymptotically stable.

In order to further prove that $\mathbf{x}_{1}=0, \mathbf{x}_{2}=0$ is the global unique equilibrium point of (37); it is needed to use Theorem 3.2 of [19]. And we can obtain that the system (37) uniformly converges to the origin in a limited time.

In order to extract the virtual control derivative signal without tedious calculation in procedure of the backstepping attitude controller design, a rapid-convergent nonlinear differentiator (RCND) is designed as follows, according to Lemma 9and Theorem 1 of [20]:

$$
\begin{gathered}
\dot{\mathbf{z}}_{1}=\mathbf{z}_{2}, \\
\kappa^{2} \dot{\mathbf{z}}_{2}=-\mathbf{R}_{1}^{1}\left(\mathbf{z}_{1}-\nu\right)-\mathbf{R}_{1}^{2} \operatorname{sig}\left(\mathbf{z}_{1}-\nu\right)^{\varepsilon /(2-\varepsilon)}-\mathbf{R}_{2}^{1} \kappa \mathbf{z}_{2} \\
-\mathbf{R}_{2}^{2} \operatorname{sig}\left(\kappa \mathbf{z}_{2}\right)^{\varepsilon}
\end{gathered}
$$

where $\mathbf{z}_{1}, \mathbf{z}_{2} \in R^{n}$ are the states of the differentiator, $v \in R^{n}$ is a known vector field, and it has almost continuous second derivative everywhere. $\kappa=\operatorname{diag}\left\{\kappa_{1}, \ldots, \kappa_{n}\right\}>0,0<\varepsilon<1$ are, respectively, the perturbation parameter and constant to be designed.

It is crucial to stress that, according to Lemma 9 and (41), we conclude that $\left\|\mathbf{z}_{1}-v\right\| \rightarrow 0,\left\|\mathbf{z}_{2}-\dot{\nu}\right\| \rightarrow 0$ come into existence in a limited time.

Remark 10. The chattering phenomenon of the differential estimation of the proposed RCDN caused by the symbol function is fully weakened by introducing the perturbation parameter. At the same time, the dynamic performance of differentiator is improved by introducing the linear part. Furthermore, because of the combination of linear differentiator and nonlinear differentiator, the proposed RCDN has the characteristics of strong robustness and rapid convergence.

4.2.2. Attitude Controller Design. To give a clear idea of the controller design procedure, the following steps are given.

Step 1. The task of this step is to design an ideal virtual angular rate command under the attitude angle loop (8) existing disturbance $\mathbf{D}_{\xi}$.
Consider the attitude angle loop dynamics (8) and define the attitude error as follows:

$$
\mathbf{Z}_{\xi}=\xi-\xi_{c}
$$

where $\xi_{c}=\left(\phi_{c}, \theta_{c}, \psi_{c}\right)^{T}$ are the desired attitude angles which are computed by (31).

Based on (8), the derivative of $\mathbf{Z}_{\xi}$ is given by

$$
\dot{\mathbf{Z}}_{\xi}=\mathbf{W}\left(\mathbf{Z}_{\Omega}+\Omega^{d}\right)+\mathbf{D}_{\xi}-\dot{\xi}_{c}
$$

where $\mathbf{Z}_{\Omega}=\Omega-\Omega^{d}$ is the angular rate tracking error, $\Omega^{d}$ is the ideal angular rate command, and it will be defined later.

We design the virtual angular rate command $\Omega^{d}$ as

$$
\Omega^{d}=W^{-1}\left(-\mathbf{C}_{\xi} \mathbf{Z}_{\xi}-\widehat{\mathbf{D}}_{\xi}+r_{\xi}+\dot{\xi}_{c}\right)
$$

where $\mathbf{C}_{\xi}$ is a diagonal positive definite matrix to be designed, $\widehat{\mathbf{D}}_{\xi}$ is the reconstruction value of the unknown disturbance $\mathbf{D}_{\xi}, r_{\xi}$ is a robust controller used to reduce the effect of reconstruction error on the control performance, and its structure is given by

$$
r_{\xi}=-\tau_{\xi} \mathbf{Z}_{\xi}
$$

where $\tau_{\xi}$ is a positive constant to be designed.

We choose the Lyapunov function $V_{1}=(1 / 2) \mathbf{Z}_{\xi}^{T} \mathbf{Z}_{\xi}$. Then, based on (43), (44), and (45), the time derivative of $V_{1}$ is given by

$$
\begin{aligned}
\dot{V}_{1} & =\mathbf{Z}_{\xi}^{T}\left[\mathbf{W}\left(\mathbf{Z}_{\Omega}+\Omega^{d}\right)+\mathbf{D}_{\xi}-\dot{\xi}_{c}\right] \\
& =\mathbf{Z}_{\xi}^{T}\left[-\mathbf{C}_{\xi} \mathbf{Z}_{\xi}+\mathbf{W} \mathbf{Z}_{\Omega}+\mathbf{D}_{\xi}-\widehat{\mathbf{D}}_{\xi}+r_{\xi}\right] \\
& \leq C_{\xi}^{-}\left\|\mathbf{Z}_{\xi}\right\|^{2}+\mathbf{Z}_{\xi}^{T} \mathbf{W} \mathbf{Z}_{\Omega}+\frac{\left\|\mathbf{e}_{\mathbf{D}_{\xi}}\right\|^{2}}{4 \tau_{\xi}}
\end{aligned}
$$

where $C_{\xi}^{-}=\lambda_{\min }\left(\mathbf{C}_{\xi}\right)$ is the smallest eigenvalue of $\mathbf{C}_{\xi}, \mathbf{e}_{\mathbf{D}_{\xi}}=$ $\mathbf{D}_{\xi}-\widehat{\mathbf{D}}_{\xi}$ is the reconstruction error of the disturbance of the attitude angle loop, $\mathbf{Z}_{\xi}^{T} \mathbf{W} \mathbf{Z}_{\Omega}$ is a coupling term, and it will be disposed in the next step.

Thus, from (46), we conclude that attitude angles $\xi$ can track the desired attitude angles $\xi_{c}$ when $\left\|\mathbf{Z}_{\Omega}\right\| \rightarrow 0$ and $\left\|\mathbf{Z}_{\xi}\right\|$ are bounded.

Step 2. The task of this step is to design control torque $\tau^{b}$ and achieve $\Omega$ asymptotical tracking $\Omega^{d}$ under the angular rate loop (9) existing composite disturbance $\mathbf{D}_{\Omega}$. At the same time, consider that the differential of the ideal virtual angular rate command can not be measured.

Taking the time derivative of the angular rate tracking error $\mathbf{Z}_{\Omega}=\Omega-\Omega^{d}$ and substituting (9) into it, yields that

$$
\dot{\mathbf{Z}}_{\Omega}=\mathbf{J}^{-1}(-\Omega \times \mathbf{J} \Omega)-\mathbf{J}^{-1} \mathbf{G}_{a}+\mathbf{J}^{-1} \tau^{b}+\mathbf{D}_{\Omega}-\dot{\Omega}^{d} .
$$


TABLE 1: Six-rotor UAV model parameters.

\begin{tabular}{lcccccccccc}
\hline Parameter & $m$ & $g$ & $l_{1}$ & $l_{2}$ & $l_{3}$ & $\alpha$ & $\mathbf{J}$ & $J_{r}$ & $b$ \\
\hline Value & 1.7 & 9.81 & 281.5 & 287.6 & 40.08 & 45 & $\operatorname{Diag}\{0.0019,0.0019,0.031\}$ & $3.357 \times 10^{-5}$ & $3.357 \times 10^{-5}$ & $3.357 \times 10^{-5}$ \\
Units & $\mathrm{Kg}$ & $\mathrm{m} / \mathrm{s}^{2}$ & $\mathrm{~mm}$ & $\mathrm{~mm}$ & $\mathrm{~mm}$ & $\mathrm{deg}$ & $\mathrm{Kg} \cdot \mathrm{m}^{2}$ & $\mathrm{Kg} \cdot \mathrm{m}^{2}$ & $\mathrm{~N} \mathrm{~s}^{2} / \mathrm{rad}^{2}$ & $\mathrm{Nm} \mathrm{s}^{2} / \mathrm{rad}^{2}$ \\
\hline
\end{tabular}
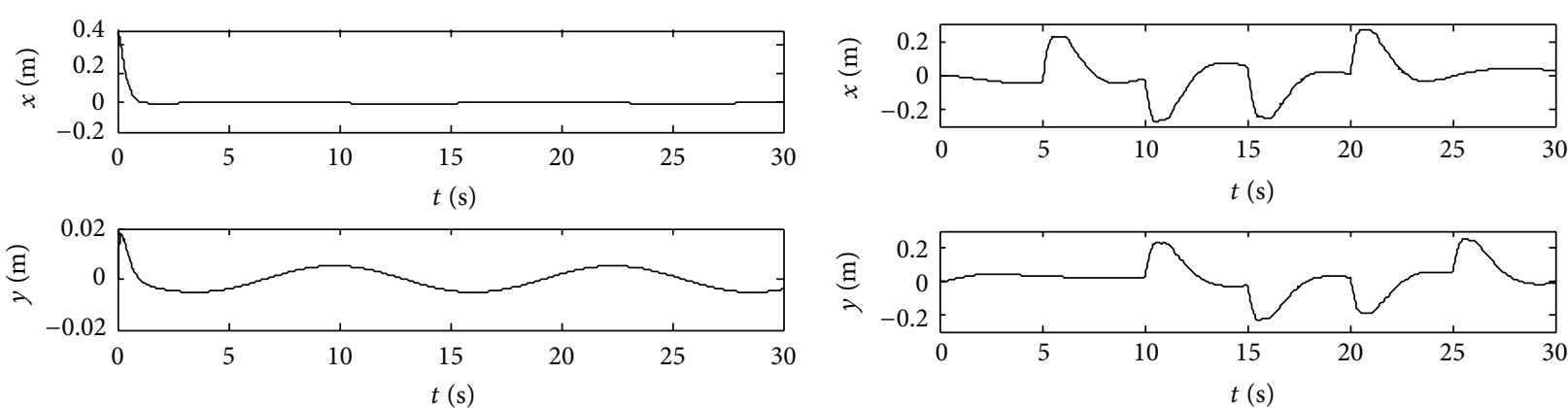

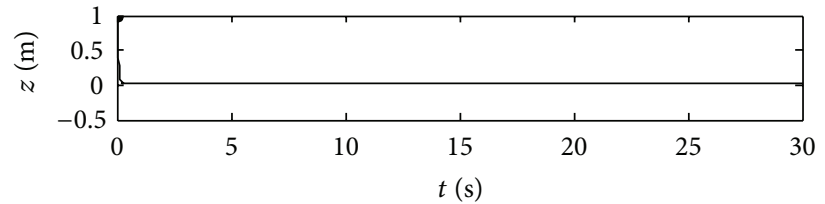

(a) Vertical helix flight

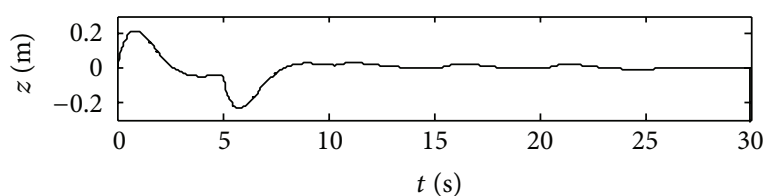

(b) Horizontal rectangle flight

FIGURE 5: Trajectory tracking errors of $x, y, z$ orientation.

In order to achieve $\mathbf{Z}_{\Omega}$ stability and eliminate the coupling term of (46), the control torques are designed as

$$
\begin{aligned}
\tau^{b}=\mathbf{J}[ & -\mathbf{C}_{\Omega} \mathbf{Z}_{\Omega}-\mathbf{W}^{T} \mathbf{Z}_{\xi}-\mathbf{J}^{-1}(-\Omega \times \mathbf{J} \Omega) \\
& \left.+\mathbf{J}^{-1} \mathbf{G}_{a}+\widehat{\hat{\Omega}}^{d}-\widehat{\mathbf{D}}_{\Omega}+r_{\Omega}\right]
\end{aligned}
$$

where $\mathbf{C}_{\Omega}$ is a positive definite parameter matrix to be designed, $\widehat{\mathbf{D}}_{\Omega}$ is the reconstruction value of the unknown composite disturbance $\mathbf{D}_{\Omega}$, and $\widehat{\dot{\Omega}}^{d}$ and $\Omega^{d}$ are, respectively, the angular rate differential estimation vector (the output) and the input of the RCDN. Similar to $r_{\xi}, r_{\Omega}$ is also a robust controller, and its structure is given by

$$
r_{\Omega}=-\tau_{\Omega} \mathbf{Z}_{\Omega},
$$

where $\tau_{\Omega}$ is a positive constant to be designed.

Aiming at (47) and considering (43), we choose the candidate compound Lyapunov function $V_{2}=V_{1}+(1 / 2) \mathbf{Z}_{\Omega}^{T} \mathbf{Z}_{\Omega}$. Then, taking the time derivative of $V_{2}$, and according to the (46)-(49) and Young inequality, we have

$$
\begin{aligned}
\dot{V}_{2} & =\dot{V}_{1}+\mathbf{Z}_{\Omega}^{T}\left[\mathbf{J}^{-1}(-\Omega \times \mathbf{J} \Omega)-\mathbf{J}^{-1} \mathbf{G}_{a}+\mathbf{J}^{-1} \tau_{a}+\mathbf{D}_{\Omega}-\dot{\Omega}^{d}\right] \\
& =\dot{V}_{1}+\mathbf{Z}_{\Omega}^{T}\left[-\mathbf{C}_{\Omega} \mathbf{Z}_{\Omega}-\mathbf{W}^{T} \mathbf{Z}_{\xi}+\hat{\dot{\Omega}}^{d}-\dot{\Omega}^{d}+\mathbf{D}_{\Omega}-\widehat{\mathbf{D}}_{\Omega}+r_{\Omega}\right] \\
& \leq \sum_{i=\xi, \Omega}\left(C_{i}^{-}\left\|\mathbf{Z}_{i}\right\|^{2}+\frac{\left\|\mathbf{e}_{\mathbf{D}_{i}}\right\|^{2}}{2 \tau_{i}}\right)+\frac{\left\|\mathbf{e}_{f}\right\|^{2}}{2 \tau_{\Omega}},
\end{aligned}
$$

where $C_{\Omega}^{-}=\lambda_{\min }\left(\mathbf{C}_{\Omega}\right)$ is the smallest eigenvalue of $\mathbf{C}_{\Omega}, \mathbf{e}_{\mathbf{D}_{\Omega}}=$ $\mathbf{D}_{\Omega}-\widehat{\mathbf{D}}_{\Omega}$ is the reconstruction error of the disturbance of the angular rate loop, and $\mathbf{e}_{f}=\widehat{\dot{\Omega}}^{d}-\dot{\Omega}^{d}$ is the estimation error of the RCDN.

4.2.3. Stability Analysis. Before proceeding with the stability analysis of this section, we recall a useful existing lemma presented in [21].

Lemma 11. If there exist constants $\alpha_{1}, \alpha_{2}, \alpha_{3}, \gamma_{1} \in K_{\infty}, \varsigma_{1}>0$ and a smooth function $V: R^{n} \rightarrow R^{+}$such that the arbitrary bounded input u satisfy

$$
\begin{gathered}
\alpha_{1}(\|x\|) \leq V(x) \leq \alpha_{2}(\|x\|), \\
\dot{V}(x) \leq-\alpha_{3}(\|x\|)+\gamma_{1}\left(\|u\|_{[0, t)}\right)+\varsigma_{1} .
\end{gathered}
$$

Then, the system $\dot{\mathbf{x}}=f(\mathbf{x}, \mathbf{u})$ is input to state practically stability (ISpS) in the interval $[0, t)$.

Theorem 12. Given the rotational dynamics of the six-rotor $U A V$ as described in (8) and (9) with a control strategy shown in Figure 3 and the SOSMDO given by (15), the attitude angle loop controller given by (44) and the angular rate loop controller given by (48), the tracking errors $\mathbf{Z}_{\xi}$ and $\mathbf{Z}_{\Omega}$ are input to state practical stability (ISpS) with respect to $\mathbf{e}_{\mathbf{D}_{\xi}}, \mathbf{e}_{\mathbf{D}_{\Omega}}$, and $\mathbf{e}_{f}$ in 

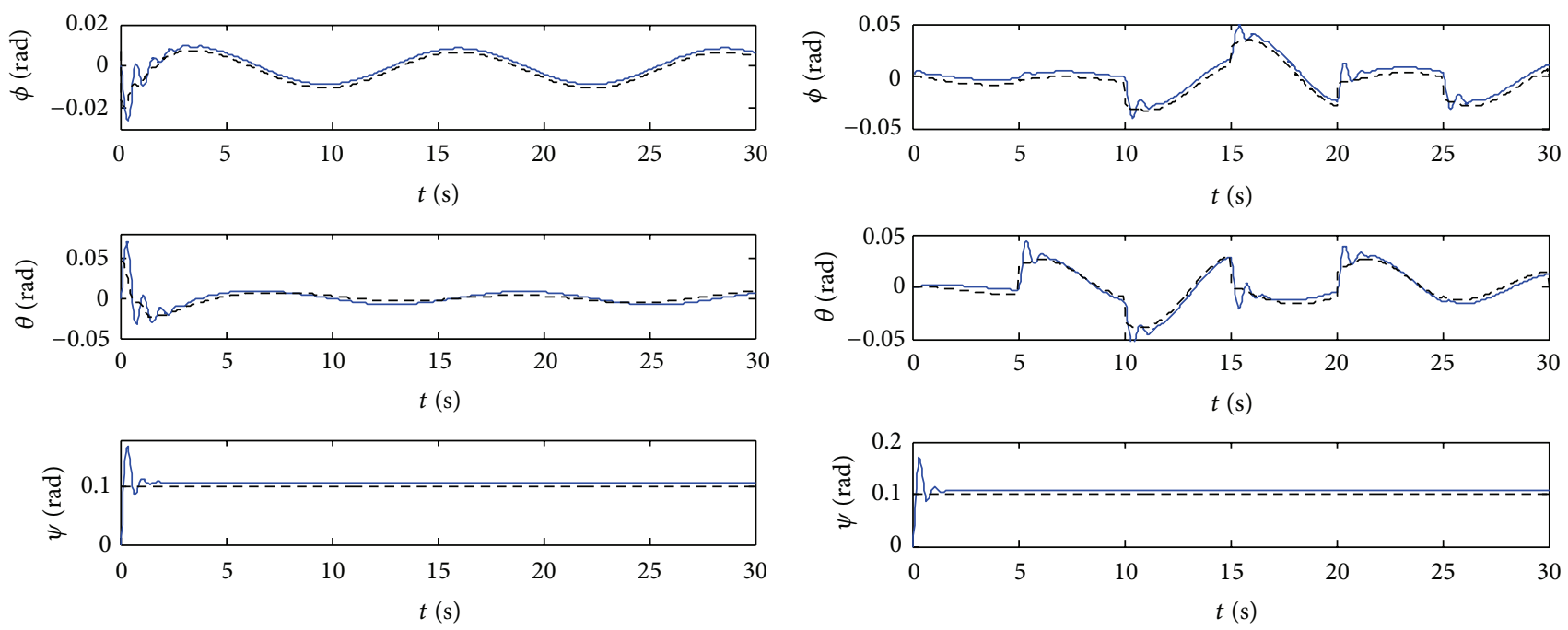

(a) Vertical helix flight

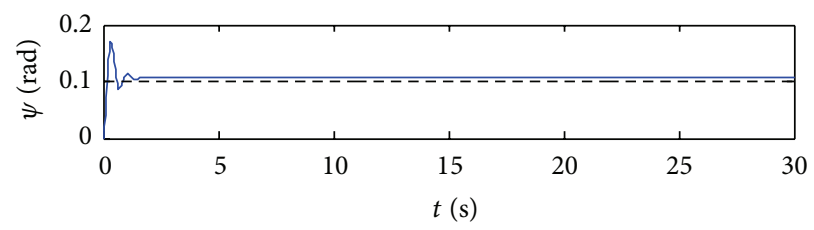

(b) Horizontal rectangle flight

FIGURE 6: Attitude angles response (solid line) and the desired attitude angles for tracking the different reference trajectory (dashed line).

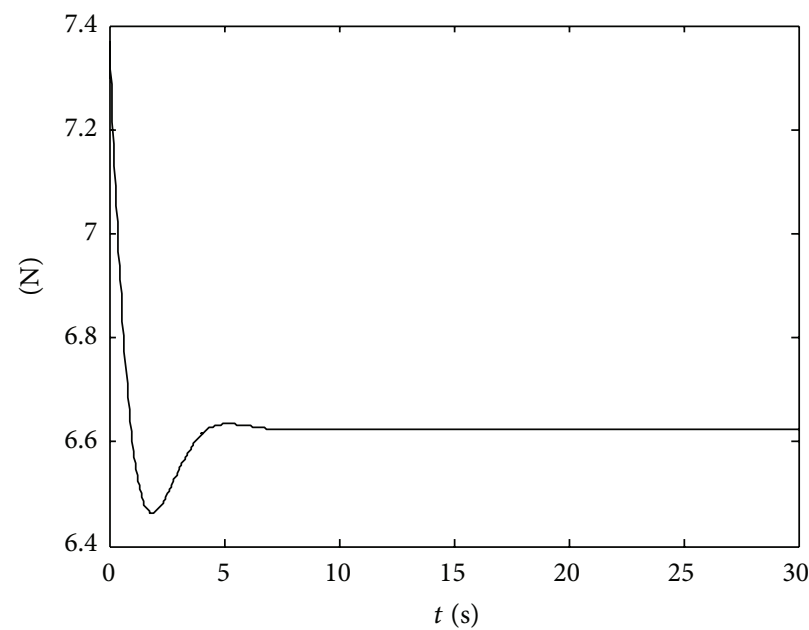

(a) Vertical helix flight

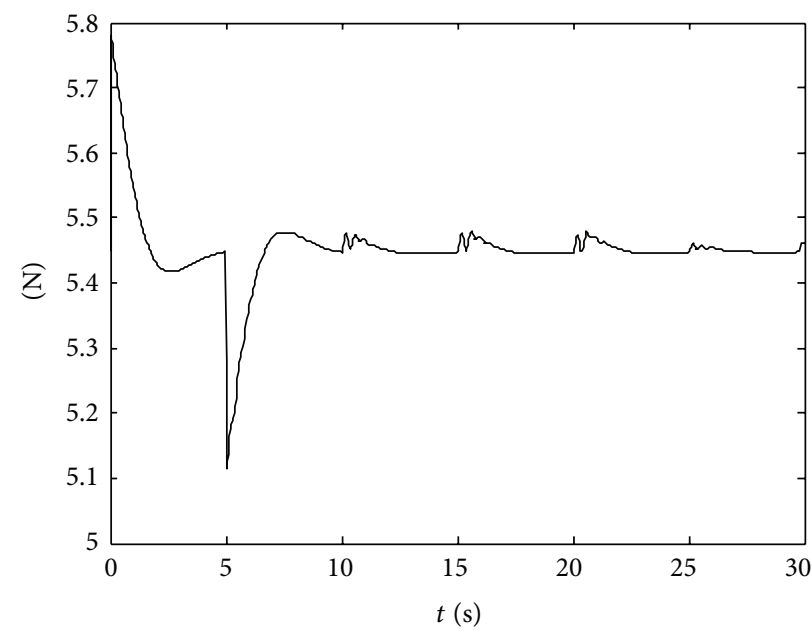

(b) Horizontal rectangle flight

Figure 7: Control thrust.

the interval $[0, t)$. This means that there exist a KL function $\beta_{1}$, a class $K_{\infty}$ function $\alpha_{1}, \gamma_{1}$, and $\gamma_{2}$ such that

$$
\begin{array}{r}
\alpha_{1}\left(\left\|\mathbf{Z}_{i}\right\|\right) \leq \beta_{1}\left(\left\|\mathbf{Z}_{i}\left(t_{0}\right)\right\|, t\right)+\gamma_{1}\left(\left\|e_{D}\right\|_{\left[t_{0}, t\right)}\right)+\gamma_{2}\left(\left\|e_{f}\right\|_{\left[t_{0}, t\right)}^{2}\right) \\
t \geq t_{0},
\end{array}
$$

where $\left\|e_{D}\right\|=\max _{i=\xi, \Omega}\left(\left\|e_{D_{i}}\right\|^{2}\right)$.

Proof. According to (50), we have

$$
\dot{V}_{2} \leq-\Upsilon V_{2}+\varsigma_{1}\left\|e_{D}\right\|+\varsigma_{2}\left\|e_{f}\right\|^{2},
$$

where $\Upsilon=\min _{i=\xi, \Omega}\left(C_{i}^{-}\right), \varsigma_{1}=\max _{i=\xi, \Omega}\left(1 / 2 \lambda_{i}\right), \varsigma_{2}=1 / 2 \lambda_{\Omega}$.

From Theorem 6, it is known that there exists a constant $\delta>0$ which makes $\left\|e_{D}\right\| \leq \delta$ established. Therefore, we can get the conclusion that the Lyapunov fuction $V_{2}$ satisfies the Lemma 11. And thus, the validity of Theorem 8 is proved.

\section{Simulation Results and Discussion}

In this section, in order to verify the validity and efficiency of the technique proposed in this paper, simulations of two typical trajectory tracking tasks are performed on Matlab/Simulink. 

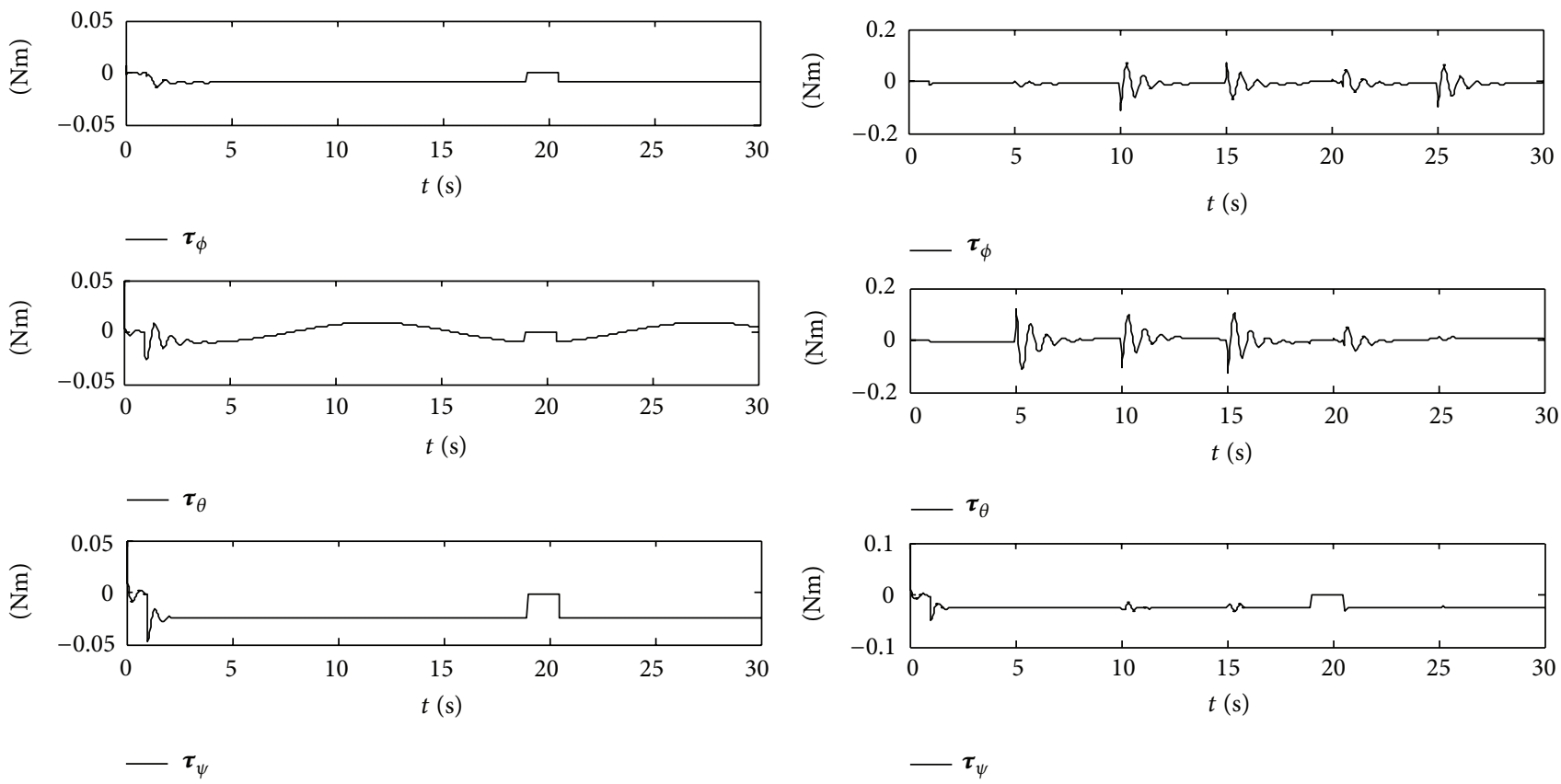

(a) Vertical helix flight

(b) Horizontal rectangle flight

Figure 8: Control torques.
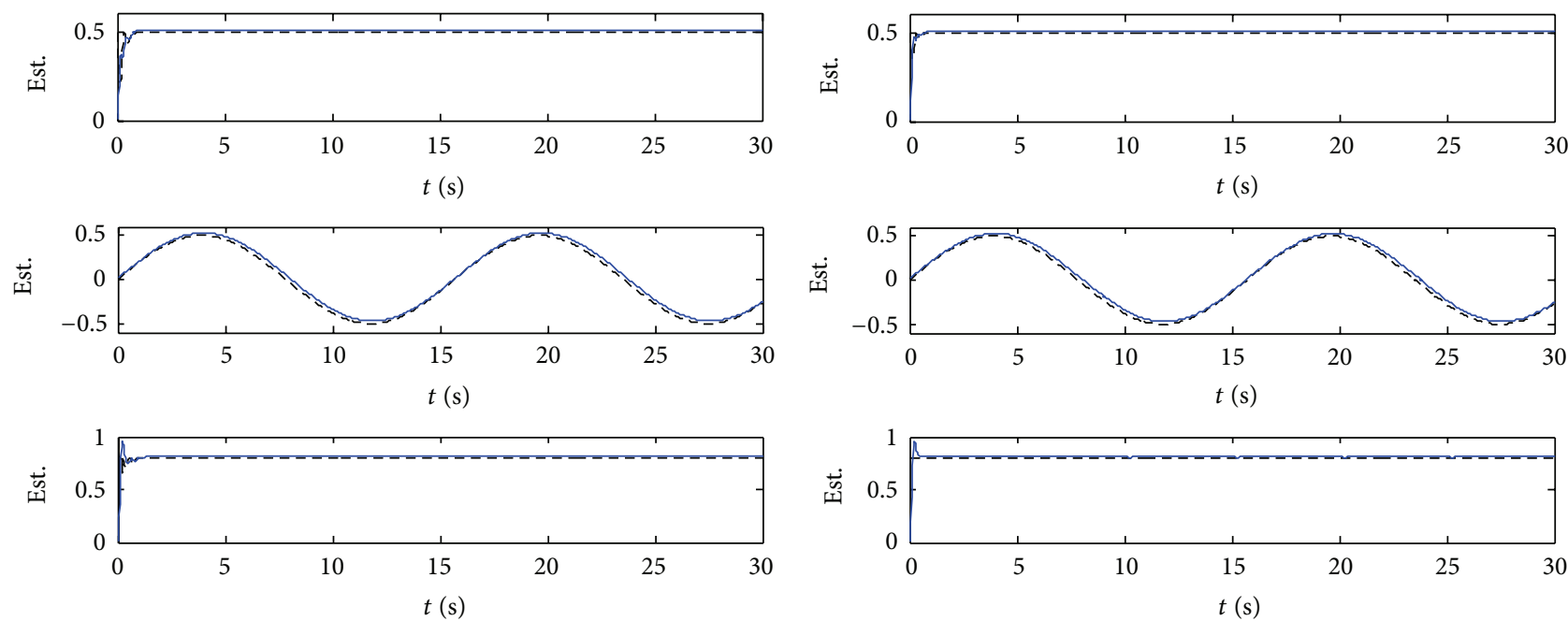

(a) Vertical helix flight

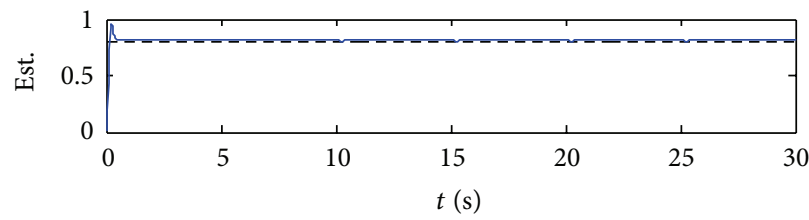

(b) Horizontal rectangle flight

FiguRE 9: Disturbance reconstruction values (solid line) and the real disturbance (dashed line).

The six-rotor UAV model parameters are taken as listed in Table 1.

In view of the limitations of real measuring device, the sampling time is fixed to $\Delta t=0.02 \mathrm{~s}$. In the simulation, the initial positions and attitude angles are $\mathbf{p}_{0}=(0,0,0)^{T}$, $\xi_{0}=(0,0,0)^{T}$, respectively, and so are linear and angular velocities, respectively. And we choose the composite disturbance input $\mathbf{D}_{\Omega}(t)=[0.5 \cos (r), 0.5 \sin (0.4 t), 0.5 \cdot \cos (p)+$ $0.3]^{T}$.
Experiment 1. The first task is to track a vertical helix trajectory which is defined as

$$
\begin{gathered}
x_{c}=0.5 \cos (0.5 t), \\
y_{c}=0.5 \sin (0.5 t), \\
z_{c}=1+0.1 t, \quad \psi_{c}=0.1 \mathrm{rad} .
\end{gathered}
$$

The position controller and attitude controller parameters in simulations are fixed at $\mathbf{K}_{r}=\operatorname{diag}\{0.5,0.5,0.5\}$, 

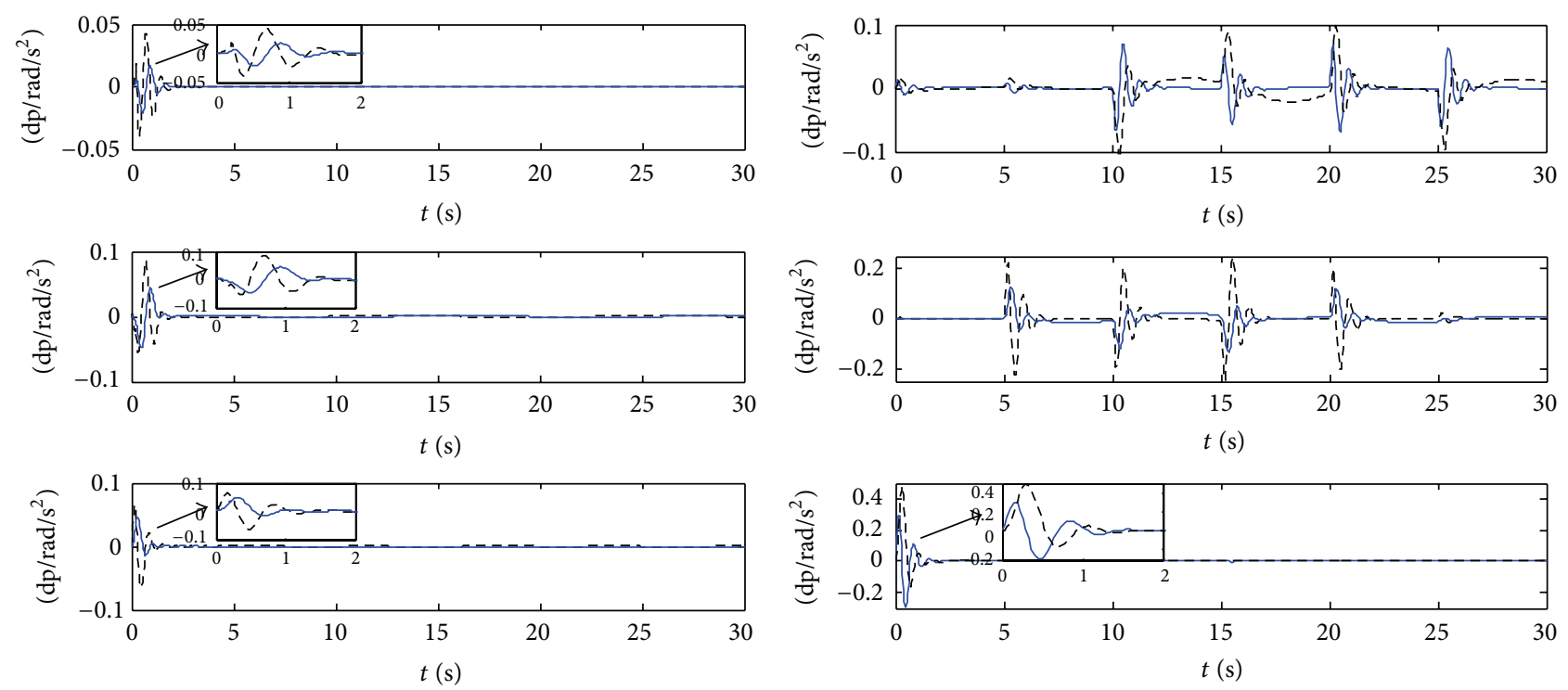

(a) Vertical helix flight

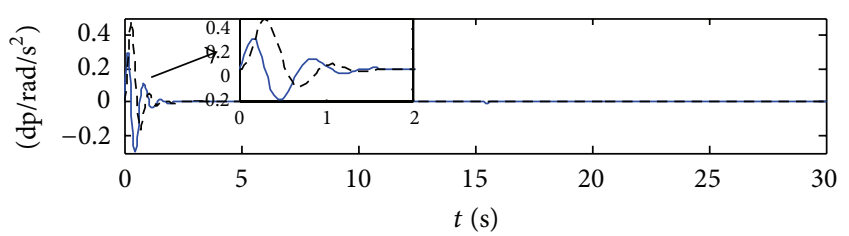

(b) Horizontal rectangle flight

FIgURE 10: Angular rate derivative estimation values using the proposed method in this paper (solid line) and the method in [13] (dashed line).

$\mathbf{K}_{i}=\operatorname{diag}\{0.2,0.2,0.2\}, \Lambda=\operatorname{diag}\{1,1,1\}, \mathbf{C}_{\xi}=\operatorname{diag}\{2,2,2\}$, $\mathrm{C}_{\Omega}=\operatorname{diag}\{5,5,5\}, \tau_{\xi}=15, \tau_{\Omega}=45$. The SOSMDO parameter is chosen as $\Gamma_{\Omega}=\operatorname{diag}\{1.2,1.0,1.5\}$, and the RCND parameters are chosen as $\kappa=\operatorname{diag}\{0.01,0.15,0.4\}, \mathbf{R}_{1}^{1}=4 \mathbf{I}_{3 \times 3}$, $\mathbf{R}_{1}^{2}=1.5 \mathbf{I}_{3 \times 3}, \mathbf{R}_{2}^{1}=\mathbf{R}_{2}^{2}=0.5 \mathbf{I}_{3 \times 3}$.

Experiment 2. The second task is to track a horizontal rectangle trajectory given by

$$
\begin{aligned}
& x_{c}= 0.8(t-5) \mathrm{fsg}(t, 5,10)+4 \mathrm{fsg}(t, 10,15) \\
&+0.8(20-t) \mathrm{fsg}(t, 15,20), \\
& y_{c}= 0.6(t-10) \mathrm{fsg}(t, 10,15)+3 \mathrm{fsg}(t, 15,20) \\
&+0.6(25-t) \mathrm{fsg}(t, 20,25), \\
& z_{c}=0.6 t \mathrm{fsg}(t, 0,5)+3 \mathrm{fsg}(t, 5,30), \quad \psi_{c}=0.1 \mathrm{rad},
\end{aligned}
$$

where fsg is an interval function and is expressed as $\mathrm{fsg}(x, a$, $b)=(\operatorname{sign}(x-a)+\operatorname{sign}(b-x)) / 2$.

In Experiment 2, the position controller parameters, attitude controller parameters, SOSMDO parameter, and the RCND parameters are chosen as same as Experiment 1.

Simulation results are presented in Figures 4, 5, 6, 7, 8, 9 , and 10 which depict the $3 \mathrm{D}$ flight trajectory, the trajectory tracking errors of $x, y, z$ orientation, the response of the attitude angles, control thrust and torques, disturbance reconstruction values and estimation values of the angular rate derivative, respectively.

Figures 4 and 6 depict the performance of trajectory tracking and the attitude angles response, respectively. They verify the proposed control strategy which has the same efficiency to track different trajectory types. In addition to this, as expected, the proposed trajectory tracking control strategy is capable of making the six-rotor UAV with aerodynamic moments, external disturbance and parameter uncertainty input track the desired trajectory in a satisfactory way, seeing Figures 4 and 5 and guaranteeing the attitude angles tracking the desired attitude angles in an appropriate scope, and seeing Figure 6. At the same time, from Figure 5, we can evidently see that the maximal trajectory tracking error does not exceed 0.2 meter which is allowed in the actual flight. So, the actual flight trajectory with disturbance compensation closely follows the desired trajectory even under the composite disturbances. So, it is obvious that the control law performs better with the disturbance reconstruction and compensation under the composite disturbances inputs.

From Figure 9, we can get the conclusion that the proposed SOSMDO can reconstruct the disturbance accurately. Thus, the correctness and effectiveness of the proposed SOSMDO are verified through this result.

Moreover, compared with the method of [13], the proposed RCND in this paper has the characteristics of faster convergence and stronger robustness which can be demonstrated by Figure 10 .

\section{Conclusions}

(1) Aiming at the potential inadequacies, such as smaller payload capacity, lack of hardware redundancy, and anticrosswind capability of the quad-rotor, a novel six-rotor UAV and its mathematical model are developed in this paper. An SOSMDO is also proposed to reconstruct the disturbance of the rotational dynamics. The correctness and effectiveness of the proposed SOSMDO are verified by the simulation results. 
(2) The proposed trajectory tracking control strategy is capable of making the six-rotor UAV track the desired trajectory in a satisfactory way. The maximal trajectory tracking error does not exceed 0.2 meter, which is allowed in the actual flight. And the results also show that this control strategy can make the six-rotor UAV track the desired attitude angles very well.

(3) Compared with the approach of [13], the proposed RCND in this paper has the characteristics of faster convergence and stronger robustness.

(4) Based on the proposed control law, how to design the flight controller for the six-rotor UAV and achieve the objective of practical flight is our future works.

\section{Acknowledgments}

This study was cosupported by Specialized Researched Fund for the Doctoral Program of Higher Education of China (no. 20113218110013), Funding of Jiangsu Innovation Program for Graduate Education (no. CXLX11_0200), the National Natural Science Foundation of China (no. 60674100), the Industry-academic Joint Technological Innovations Fund Project of Jiangsu Province, China (no. BY2012018), and the Natural Science Foundation of Shandong Province, China (no. ZR2012FQ030).

\section{References}

[1] E. Altuğ, J. P. Ostrowski, and C. J. Taylor, "Control of helicopter using dual camera visual feedback," International Journal of Robotics Research, vol. 24, no. 5, pp. 329-341, 2005.

[2] H. Bouadi, M. Bouchoucha, and M. Tadhine, "Sliding mode control based on backstepping approach for an UAV typequadrotor," International Journal of Applied Mathematics \& Computer Sciences, vol. 4, no. 1, pp. 12-17, 2008.

[3] A. A. Mian and D. B. Wang, "Modeling and backstepping-based nonlinear control strategy for a 6 DOF quadrotor helicopter," Chinese Journal of Aeronautics, vol. 21, no. 3, pp. 261-268, 2008.

[4] Z. Zuo, "Trajectory tracking control design with commandfiltered compensation for a quadrotor," IET Control Theory \& Applications, vol. 4, no. 11, pp. 2343-2355, 2010.

[5] I. H. Choi and H. C. Bang, "Adaptive command filtered backstepping tracking controller design for quadrotor unmanned aerial vehicle," Proceedings of the Institution of Mechanical Engineers G, vol. 226, no. 5, pp. 483-497, 2012.

[6] Z. Yang, X. N. Huang, and Q. L. Li, "A novel multi-rotor aircraft with symmetrical layout," Chinese Patent: CN202071985U, 2011.

[7] Z. Yang, X. N. Huang, and Q. L. Li, “Transmission lines inspection system based on multi-rotor unmanned aerial vehicle," Chinese Patent: CN202042825U, 2011.

[8] B. Wang, P. Shi, H. R. Karimi, J. Wang, and Y. Song, "Fuzzy variable structure control for uncertain systems with disturbance," Mathematical Problems in Engineering, vol. 2012, Article ID 105074, 12 pages, 2012.

[9] R. Yang, P. Shi, G.-P. Liu, and H. Gao, "Network-based feedback control for systems with mixed delays based on quantization and dropout compensation," Automatica, vol. 47, no. 12, pp. 2805-2809, 2011.
[10] A. Das, K. Subbarao, and F. Lewis, "Dynamic inversion with zero-dynamics stabilisation for quadrotor control," IET Control Theory \& Applications, vol. 3, no. 3, pp. 303-314, 2009.

[11] D. Lee, H. J. Kim, and S. Sastry, "Feedback linearization versus adaptive sliding mode control for a quadrotor helicopter," International Journal of Control, Automation and Systems, vol. 7, no. 3, pp. 419-428, 2009.

[12] Q. Zhou, P. Shi, H. Liu, and S. Xu, "Neural-network-based decentralized adaptive output-feedback control for large-scale stochastic nonlinear systems," IEEE Transaction on Systems, Man and Cybernetics, Part B, vol. 42, no. 6, pp. 1608-1619, 2012.

[13] G. D. Yang and Y. Zhou, "Analysis and simulation of steady-state performance in nonlinear tracking differentiator," Electro-Optic Technology Application, vol. 25, no. 5, pp. 80-82, 2010.

[14] H. Goldstein, Classical Mechanics, Addison-Wesley Publishing, Reading, Mass, USA, 2nd edition, 1980.

[15] L. Besnard, Y. B. Shtessel, and B. Landrum, "Control of a quadrotor vehicle using sliding mode disturbance observer," in Proceedings of the American Control Conference (ACC '07), pp. 5230-5235, New York, NY, USA, July 2007.

[16] X. F. Zeng, J. Y. Wang, X. H. Wang, and T. Wang, "Design of sliding mode controller based on SMDO and its application to missile control," Acta Aeronautica et Astronautica Sinica, vol. 32, no. 5, pp. 873-880, 2011.

[17] F. Lewis, S. Jagannathan, and A. Yesildirek, Neural Network Control of Robot Manipulators and Nonlinear Systems, Taylor and Francis, London, UK, 1999.

[18] S. Ibrir, W. F. Xie, and C.-Y. Su, "Adaptive tracking of nonlinear systems with non-symmetric dead-zone input," Automatica, vol. 43, no. 3, pp. 522-530, 2007.

[19] Y. Orlov, "Finite time stability and robust control synthesis of uncertain switched systems," SIAM Journal on Control and Optimization, vol. 43, no. 4, pp. 1253-1271, 2005.

[20] X. Wang, Z. Chen, and G. Yang, "Finite-time-convergent differentiator based on singular perturbation technique," IEEE Transactions on Automatic Control, vol. 52, no. 9, pp. 1731-1737, 2007.

[21] H. K. Khalil, Nonlinear Systems, Prentice Hall, Upper Saddle River, NJ, USA, 3rd edition, 2002. 


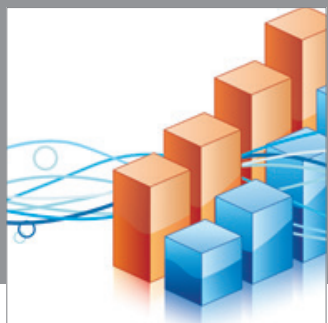

Advances in

Operations Research

mansans

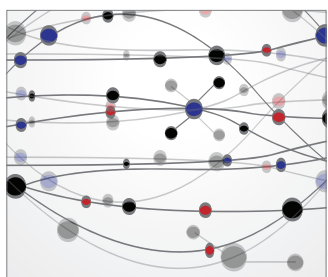

The Scientific World Journal
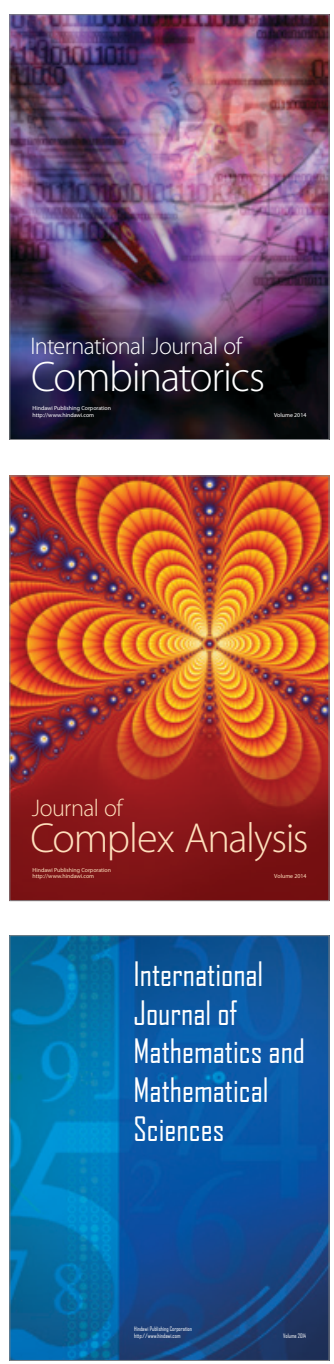
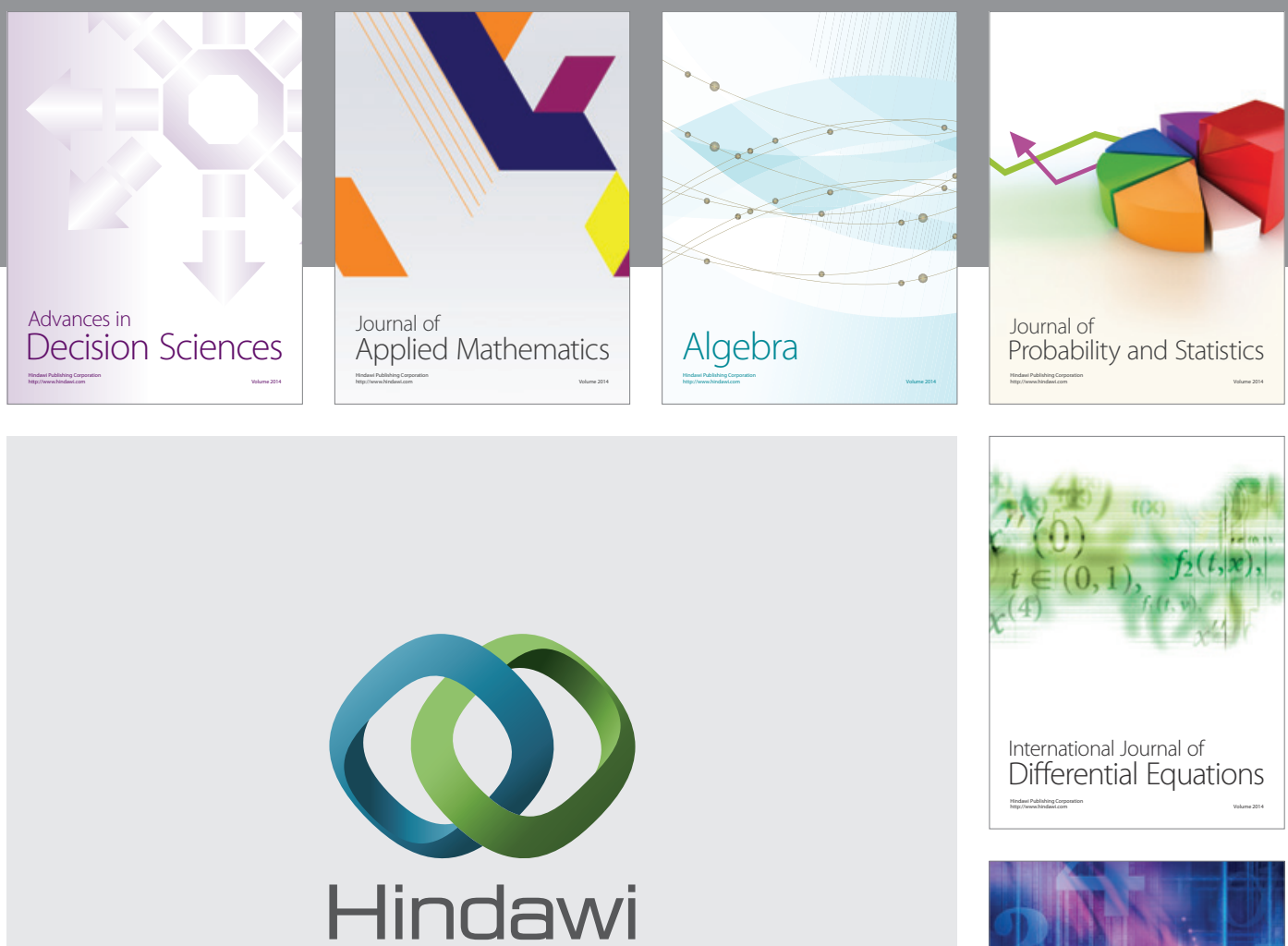

Submit your manuscripts at http://www.hindawi.com
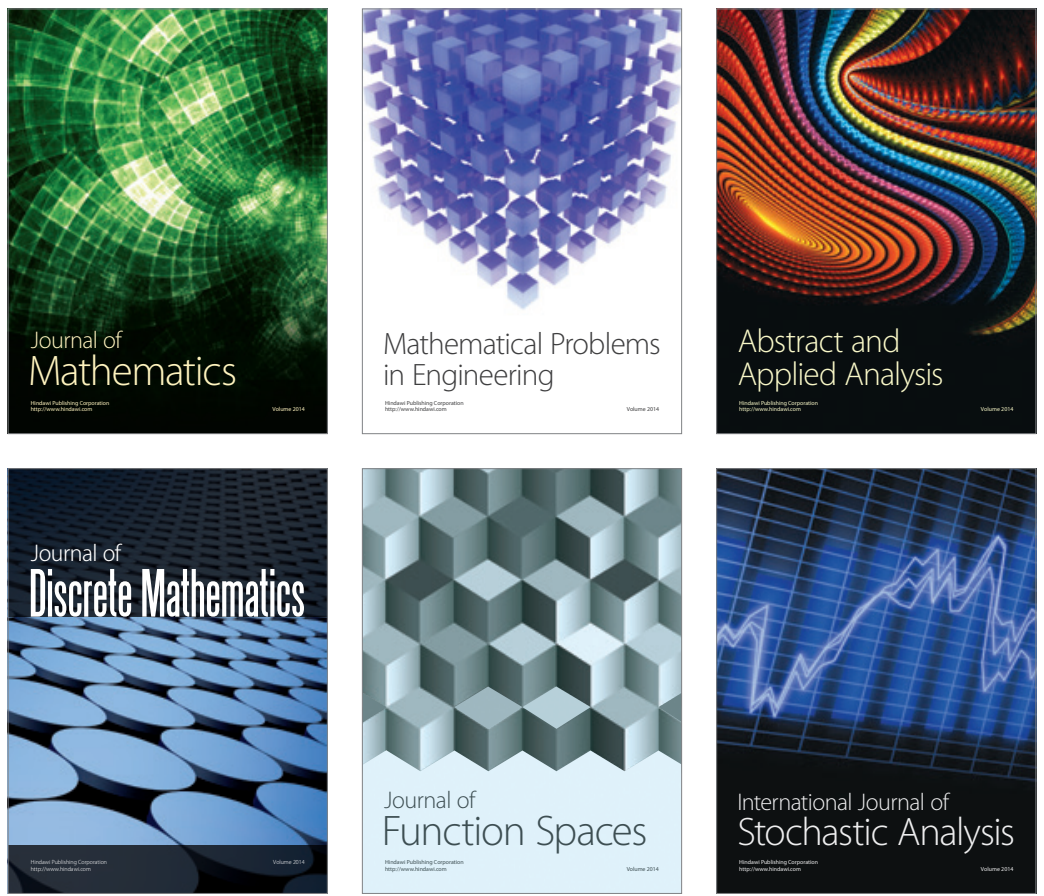

Journal of

Function Spaces

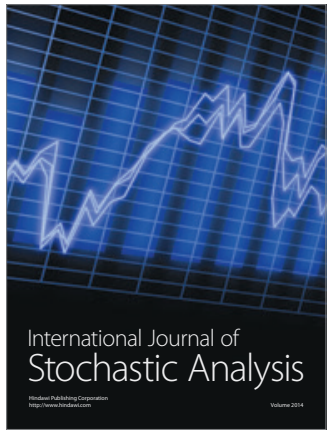

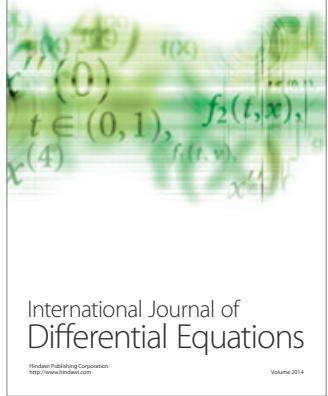
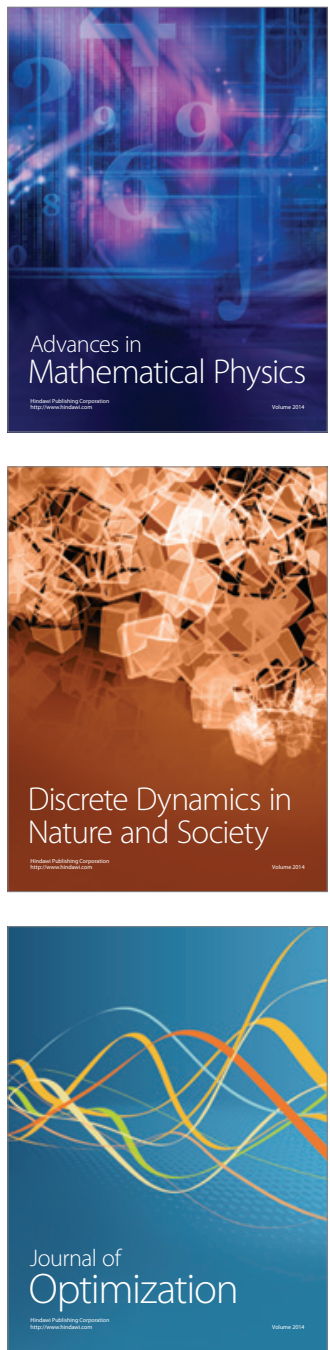